\title{
On the properties of the interstellar medium in extremely metal-poor blue compact dwarf galaxies
}

\section{GMOS-IFU spectroscopy and SDSS photometry of the double-knot galaxy HS 2236+1344 ${ }^{\star}$}

\author{
P. Lagos ${ }^{1}$, P. Papaderos ${ }^{1}$, J. M. Gomes ${ }^{1}$, A. V. Smith Castelli ${ }^{2,3}$, and L. R. Vega ${ }^{4}$ \\ ${ }^{1}$ Centro de Astrofísica da Universidade do Porto, Rua das Estrelas, 4150-762 Porto, Portugal \\ e-mail: plagos@astro.up.pt \\ 2 Instituto de Astrofísica de La Plata (CCT La Plata, CONICET, UNLP), Paseo del Bosque, B1900FWA La Plata, Argentina \\ 3 Facultad de Ciencias Astronómicas y Geofísicas, Universidad Nacional de La Plata, Paseo del Bosque, B1900FWA La Plata, \\ Argentina \\ ${ }^{4}$ Observatorio Astronómico de Córdoba, Laprida 854, 5000 Córdoba, Argentina
}

Received 29 December 2013 / Accepted 26 May 2014

\begin{abstract}
Aims. The main goal of this study is to carry out a spatially resolved investigation of the warm interstellar medium (ISM) in the extremely metal-poor blue compact dwarf (BCD) galaxy HS 2236+1344. Special emphasis is laid on analysis of the spatial distribution of chemical abundances, emission-line ratios, and the kinematics of the ISM, and to the recent star-forming (SF) activity in this galaxy. Methods. This study is based on optical integral field unit spectroscopy data from Gemini Multi-Object Spectrograph (GMOS) at the Gemini North telescope and archival Sloan Digital Sky Survey (SDSS) images. The galaxy was observed at medium spectral resolution over the spectral range from $\sim 4300 \AA$ to $7300 \AA$. The data were obtained in two different positions across the galaxy, obtaining a total $4^{\prime \prime} \times 8^{\prime \prime}$ field that encompasses most of its ISM.

Results. Emission-line maps and broad-band images obtained in this study indicate that HS 2236+1344 hosts three giant H II regions (GH IIRs). Our data also reveal some faint curved features in the BCD periphery that might be due to tidal perturbations or expanding ionized-gas shells. The ISM velocity field shows systematic gradients along the major axis of the BCD, with its southeastern and northwestern half differing by $\sim 80 \mathrm{~km} \mathrm{~s}^{-1}$ in their recessional velocity over the field of view. The $\mathrm{H} \alpha$ and $\mathrm{H} \beta$ equivalent-width distribution in the central part of HS $2236+1344$ is consistent with a very young ( $\sim 3 \mathrm{Myr}$ ) burst. Our surface photometry analysis reveals an underlying low surface brightness component with moderately red colors, which suggest that the galaxy has undergone previous star formation. We derive an integrated oxygen abundance of $12+\log (\mathrm{O} / \mathrm{H})=7.53 \pm 0.06$ and a nitrogen-to-oxygen ratio of $\log (\mathrm{N} / \mathrm{O})=-1.57 \pm 0.19$. Our results are consistent, within the uncertainties, with a homogeneous distribution of oxygen and nitrogen within the ISM of the galaxy. The high-ionization He II $\lambda 4686$ emission line is detected only in the central part of HS $2236+1344$. Similar to many BCDs with He II $\lambda 4686$ emission, HS 2236+1344 shows no Wolf-Rayet (WR) bump.
\end{abstract}

Key words. galaxies: individual: HS 2236+1344 - galaxies: dwarf - galaxies: abundances - galaxies: ISM - galaxies: star formation galaxies: photometry

\section{Introduction}

Low-mass galaxies undergoing a violent burst of star formation, such as blue compact dwarf (BCD) galaxies (Thuan \& Martin 1981; Loose \& Thuan 1986) in the local Universe and analogous objects at higher redshift (e.g., compact narrow emissionline galaxies and green peas, Guzmán et al. 1998; Cardamone et al. 2009; Amorín et al. 2012), are important testbeds of galaxy evolution. In particular, the most metal-poor of these systems (e.g., Papaderos et al. 2008; and references therein) are of special importance, since they are the closest analogs of the lowmass galaxy building blocks that are thought to have formed in the early Universe.

BCDs, the low-mass, high-compactness members of the broader class of H II galaxies (Terlevich et al. 1991; Telles

* The reduced and calibrated data cube are only available at the CDS via anonymous ftp to cdsarc.u-strasbg. fr (130.79.128.5) or via http://cdsarc.u-strasbg.fr/viz-bin/qcat?]/A+A/569/A110 et al. 1997) span a wide range in morphology and gas-phase metallicity (Kunth \& Sargent 1983; Izotov \& Thuan 1999). The majority of these systems have subsolar oxygen abundance $7.9 \lesssim 12+\log (\mathrm{O} / \mathrm{H}) \lesssim 8.4$ (Terlevich et al. 1991; Izotov \& Thuan 1999; Kunth \& Östlin 2000) and fall into the iE- or $\mathrm{nE}$ type of the classification scheme by Loose \& Thuan (1986), owing to the presence of one or several luminous star-forming (SF) regions in the central part of an old, more extended stellar low-surface brightness (LSB) host (Papaderos et al. 1996a; Telles \& Terlevich 1997; Cairós et al. 2001; Bergvall \& Östlin 2002; Gil de Paz et al. 2003; Amorín et al. 2009, see Bergvall 2012 , for a review). A smaller fraction ( $\sim 10 \%)$ of BCDs exhibit cometary morphology (iI,C in the Loose \& Thuan 1986 classification) that is due to strong ongoing SF activity at the one tip of an elongated stellar LSB "tail".

The lowest metallicity BCDs (XBCDs) or extremely metalpoor (XMP) BCDs, defined as systems with an oxygen abundance $12+\log (\mathrm{O} / \mathrm{H}) \lessgtr 7.6$ (Izotov \& Thuan 1999), are the least chemically evolved emission-line galaxies currently known. 
These systems are very scarce in the nearby Universe (Izotov \& Thuan 2007; Kniazev et al. 2004; Papaderos et al. 2008; Guseva et al. 2009; see also Skillman 2012) with only about 100 of them currently known (see Morales-Luis et al. 2011; Filho et al. 2013 , for recent compilations of literature data). The most metalpoor ones are SBS $0335-052 \mathrm{~W}(12+\log (\mathrm{O} / \mathrm{H}) \simeq 6.9 \ldots 7.12$, Izotov et al. 2005, 2009; Papaderos et al. 2006), SBS 0335-052 E $(12+\log (\mathrm{O} / \mathrm{H})=7.2 \ldots 7.3$, Izotov et al. 1997a; Papaderos et al. 2006) and $\mathrm{I} \mathrm{Zw} 18(12+\log (\mathrm{O} / \mathrm{H})=7.2$, Izotov et al. 1997b). As pointed out by Papaderos et al. (2008), BCDs/XBCDs show an intriguing connection between gas-phase metallicity, morphology, and evolutionary status, with cometary morphology being a typical characteristic of the most metal-poor systems. This, together with the low ( $\gtrsim 1 \mathrm{Gyr}$ ) luminosity-weighted age of XBCDs, suggests that most of their stellar mass has formed in the past 1-3 Gyr (Papaderos et al. 2008). Interestingly, a significant fraction $(\geq 10 \%)$ of unevolved high-redshift galaxies in the Hubble Deep Field also show cometary (also referred to as "tadpole") morphology (van den Bergh et al. 1996; Elmegreen et al. 2005, 2007; Straughn et al. 2006; Windhorst et al. 2006).

The formation process of cometary field galaxies near and far is unclear. Proposed hypotheses range from propagating SF, in the case of XBCDs (Papaderos et al. 1998), to interactions (Straughn et al. 2006) and gas instabilities in forming disks (Elmegreen et al. 2005, 2012) in the case of more massive galaxies. In fact, all these mechanisms may be at work at some level. For example, whereas $\mathrm{HI} / \mathrm{BCD}$ are spatially well separated from normal (Hubble-type) galaxies (Telles \& Maddox 2000), they usually have low-mass stellar and/or gaseous companions (e.g., Taylor et al. 1995; Noeske et al. 2001). This also applies to many XBCDs, many of which are known to reside within loose galaxy groups (Pustilnik et al. 2001a) or in galaxy pairs with a linear separation between $\sim 2 \mathrm{kpc}$ and $\sim 100 \mathrm{kpc}$ (Papaderos 2012, hereafter P12). Examples of such binary dwarf galaxies are, e.g. I Zw 18 (Lequeux \& Viallefond 1980) and SBS 0335052 (Papaderos et al. 1998; Pustilnik et al. 2001b). Recently, P12 pointed out the intriguing similarity of some XBCDs with their low-mass companions in their structural properties and evolutionary status, and interpreted this as manifestation of synchronization in the assembly history of such binary galaxies. He proposed, based on heuristic arguments, that the delayed formation of some present-day XBCDs in pairs or groups can qualitatively be explained as the result of their co-evolution within a common dark matter (DM) halo: The cumulative effect of recurrent mild interactions between co-evolving dwarfs is, according to P12, a quasi-continuous heating of their gas component and the delay of the dominant phase of their formation, in agreement with the observed low chemical abundances and young luminosityweighted ages of XBCDs.

On sub-galactic scales, observations of $\mathrm{H}$ II/BCDs also hint at a synchronous star formation history (SFH). For example, near-IR photometry and optical spectroscopy studies of UM 461 and Mrk 36 by Lagos et al. (2011) and UM 408 by Lagos et al. $(2009,2011)$ reveal that young stellar clusters (YSCs) in these galaxies have a similar age over a spatial scale of $\sim 1 \mathrm{kpc}$ (see, e.g., Telles 2010). Evidence for coeval star formation on scales of several hundred pc has also been found through photometric studies of high-luminosity BCDs with the Hubble Space Telescope (e.g., Östlin et al. 2003; Adamo et al. 2011) and surface photometry studies. For example, the subtraction of the underlying LSB host emission from surface brightness profiles of the BCD Mrk 178 yields almost flat color gradients within the starburst component, in agreement with the picture of a

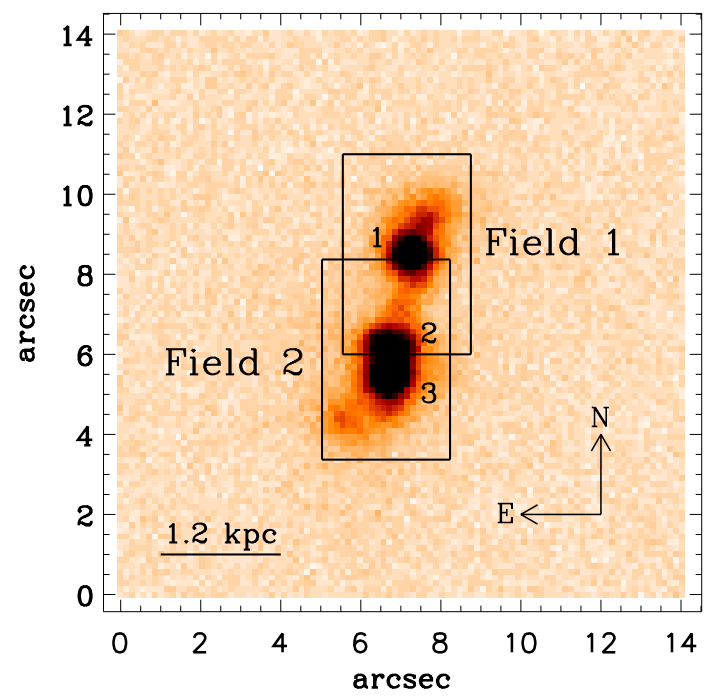

Fig. 1. $g$-band acquisition image of the galaxy HS 2236+1344. The rectangles indicate the position of the two GMOS-IFU FoV of $33^{\prime \prime} 5 \times 5^{\prime \prime}$. The three GH IIRs, resolved in this study, are indicated in the figure.

nearly coevally triggered SF episode on spatial scales of $\sim 1 \mathrm{kpc}$ (Papaderos et al. 2002).

With regard to the chemical abundance patterns of $\mathrm{HII} / \mathrm{BCDs}$, the available data suggest that the metals from previous SF events are homogeneously distributed and mixed over the interstellar medium (ISM), while freshly produced elements by massive stars remain unmixed with the warm ISM and reside in the hot gas phase (Tenorio-Tagle 1996; Kobulnicky \& Skillman 1997). The spatial constancy of the N/O ratio, observed in these galaxies, might also be attributed to efficient transport and mixing of metals (e.g., Pérez-Montero et al. 2011; Lagos et al. 2009, 2012) by hydrodynamical processes (e.g., starburst-driven flows, infall of gas), so keeping the N/O ratio constant through the ISM of the galaxies on large scales. Locally enhanced N/O ratios in some BCDs are probably explained by nitrogen enrichment through WR winds (Walsh \& Roy 1989; Kobulnicky et al. 1997), resulting in an N overabundance at the location of YSCs, in agreement with the detection of small, yet statistically significant oxygen abundance variations, attributable to chemical self-enrichment (Kunth \& Sargent 1986) even in some XBCDs (Papaderos et al. 2006).

Integral field unit (IFU) spectroscopy offers a powerful tool to study all above topical issues in $\mathrm{BCD} / \mathrm{XBCD}$ research in spatial detail (an overview of all $\mathrm{HII} / \mathrm{BCD}$ galaxies studied thus far with IFU spectroscopy is shown in Lagos \& Papaderos 2013). Our main objective in this paper is to carry out a spatially resolved investigation of the chemical abundance patterns and kinematics of the warm ISM in the nearby $(\sim 80 \mathrm{Mpc})$ XBCD galaxy HS $2236+1344$. For this, we use mediumresolution $(R \sim 4000)$ IFU data obtained with the Gemini North telescope. In Fig. 1 we show the $g$-band acquisition image of the galaxy (see Table 1 for a summary of its general properties). It can be seen that HS 2236+1344 contains three compact (regions 1-3) high-surface brightness knots or GH IIRs, and some faint arm-like features departing from its central body. These may be attributed to starburst-driven shells and/or tidally induced features, perhaps connected with an ongoing merger.

This paper is organized as follows: the observations and data reduction are discussed in Sect. 2. In Sect. 3 we presented and discuss the results. Our conclusions are summarized in Sect. 4. 
Table 1. General parameters of HS 2236+1344.

\begin{tabular}{lcc}
\hline \hline Parameter & Value & Reference \\
\hline$\alpha(\mathrm{J} 2000)$ & $22^{\mathrm{h}} 38^{\mathrm{m}} 31.1^{\mathrm{s}}$ & (a) \\
$\delta(\mathrm{J} 2000)$ & $+14^{\circ} 00^{\mathrm{m}} 30^{\mathrm{s}}$ & $(\mathrm{a})$ \\
$D(3 \mathrm{~K} C \mathrm{CMB})$ & $79.7 \mathrm{Mpc}$ & $($ a) \\
$1^{\prime \prime}(3 \mathrm{~K} \mathrm{CMB})$ & $386 \mathrm{pc}$ & $($ a) \\
$z$ & 0.020628 & $(\mathrm{~b})$ \\
$12+\log (\mathrm{O} / \mathrm{H})$ & 7.53 & $(\mathrm{~b})$ \\
$\log (\mathrm{M}(\mathrm{H} \mathrm{I})) M_{\odot}$ & $<8.35$ & $(\mathrm{c})$ \\
Other name & SDSS J223831.12+140029.7 & \\
\hline
\end{tabular}

Notes. ${ }^{(a)}$ Obtained from NED, ${ }^{(b)}$ derived from the present observations, ${ }^{(c)}$ Pustilnik \& Martin (2007).

Table 2. Observing log.

\begin{tabular}{lccc}
\hline \hline Name & Date & $\begin{array}{c}\text { Exp. time } \\
(\mathrm{s})\end{array}$ & Airmass $^{1}$ \\
& & $3 \times 1285$ & 1.068 \\
Field 1 & $2010-09-08$ & $3 \times 1285$ & 1.013 \\
\hline Field 2 & $2010-09-08$ & $3 \times 1285$ & \\
\hline
\end{tabular}

Notes. ${ }^{(1)}$ Obtained from the average values of the different exposures.

\section{Observations and data reduction}

The observations discussed in this paper were performed using the Gemini GMOS (Hook et al. 2004) and the IFU unit, hereafter GMOS-IFU (Allington et al. 2002), at Gemini North telescope, using the grating R600+_G5304 (R600) in one-slit mode with a spectral resolution of $R \sim 4000$. The GMOS-IFU in one slit mode composes a pattern of 500 hexagonal elements, each with a projected diameter of 0 ' 2 , covering a total field of view (FoV) of $3^{\prime \prime} \cdot 5 \times 5^{\prime \prime}$, plus 250 elements sampling the sky. The data were obtained at low airmass during the same night but in two different positions across the galaxy as indicated in Fig. 1 (cf. Table 2).

The data reduction was carried out using the Gemini software package version 1.9 within IRAF $^{1}$. This includes bias subtraction, flat-field correction, wavelength calibration and sky subtraction. The flux calibration was performed using the sensitivity function derived from observation of the spectrophotometric standard star $\mathrm{BD}+28 \mathrm{~d} 4211$. The $2 \mathrm{D}$ data images were transformed into $3 \mathrm{D}$ data cubes, resampled to a $0{ }^{\prime} 2$ spatial resolution, and corrected for differential atmospheric refraction using the gfcube routine. Finally, the two data cubes obtained with the grating R600 were combined into a final data cube that covers a spectral range from $\sim 4300 \AA$ to $7300 \AA$ and a FoV of $4^{\prime \prime} \times 8^{\prime \prime}$ $(20 \times 40$ spaxels). More details about the data reduction can be found in Lagos et al. (2009, 2012).

Figure 2 shows the integrated spectrum of the galaxy (over the whole FoV) and the spectra of regions 1-3, summed up over all spaxels within the areas indicated in Sect. 3.1. In this figure we label the strongest emission lines detected and used in our study. The most remarkable finding from our data is the localized He II $\lambda 4686$ emission only in region 3 (also visible on the integrated spectrum of HS 2236+1344) and a broad component in the base of some emission lines, such as $\mathrm{H} \alpha$ and [O III] $\lambda 5007$. Finally, the emission line fluxes were measured using the IRAF task fitprofs by fitting Gaussian profiles. Since most of the emission lines were measured using an automatic procedure, we as-

\footnotetext{
1 IRAF is distributed by NOAO, which is operated by the Association of Universities for Research in Astronomy Inc., under cooperative agreement with the National Science Foundation.
}

signed the value 0.0 to all spaxels with signal to noise ratio $(S / N)<3$. When this automatic procedure was not possible, the emission lines were measured manually using splot.

\section{Results and discussion}

\subsection{Emission lines, continuum and extinction structure}

We used the flux measurement procedure described previously in Sect. 2 to construct emission line maps (e.g., [S II] $\lambda 6731$,

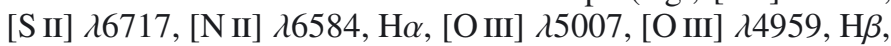
$[\mathrm{O}$ III $] \lambda 4363, \mathrm{H} \gamma$ ) within the combined FoV of $4^{\prime \prime} \times 8^{\prime \prime}$. In Fig. 3 we show the $\mathrm{H} \alpha, \mathrm{H} \alpha$ continuum, [N II] $\lambda 6584$, [S II] $\lambda 6717$, $\mathrm{EW}(\mathrm{H} \alpha)$ and the extinction $c(\mathrm{H} \beta)$ maps of the galaxy. The $\mathrm{H} \alpha$ continuum map shows that HS $2236+1344$ is composed of three GH IIRs. We designate the GH IIR that is centered on the field 1 (see Fig. 1) as region 1, while the GH IIR in the field 2 is resolved into a double peak that we labeled as regions 2 and 3 . These three GH IIRs are also resolved on the acquisition image of the galaxy (Fig. 1). We see that the structure of the ionized gas, as traced by the emission lines and the $\mathrm{H} \alpha$ and $\mathrm{H} \beta$ equivalent widths are similar, without any offset compared with the distribution of the stellar continuum (see Fig. 3).

The extinction was calculated from the emission line ratio $F(\mathrm{H} \alpha) / F(\mathrm{H} \beta)=2.76$, assuming case B recombination (Osterbrock \& Ferland 2006) at $T=2 \times 10^{4} \mathrm{~K}$ (see Table 5). The $c(\mathrm{H} \beta)$ values in Fig. 3 ranges between 0 and $\sim 0.92$ and shows an inhomogeneous pattern, with its peak value being slightly offset from that of the $\mathrm{H} \alpha$ emission. Most of the spaxels in the outer part of the galaxy show a $F(\mathrm{H} \alpha) / F(\mathrm{H} \beta)$ ratio that is close to the theoretical value, so we assumed that these regions do not suffer from intrinsic extinction. Our results support the conclusion by Cairós et al. (2009) and Lagos et al. (2009) that a constant correction term for the extinction over the whole galaxy may in some cases lead to large errors in the derivation of properties of individual regions in HII and BCD galaxies.

Finally, the observed emission line fluxes, for all the spaxels in our FoV, have been corrected for extinction using the observed Balmer decrement and the Cardelli et al. (1989) reddening curve $f(\lambda)$ as $I(\lambda) / I(\mathrm{H} \beta)=F(\lambda) / F(\mathrm{H} \beta) \times 10^{c(\mathrm{H} \beta) f(\lambda)}$. The observed $F(\lambda)$ and corrected emission line fluxes $I(\lambda)$ relative to the $\mathrm{H} \beta$ and their errors, multiplied by a factor of 100 , the observed flux of the $\mathrm{H} \beta$ emission line, the $\mathrm{EW}(\mathrm{H} \alpha)$ and $\mathrm{EW}(\mathrm{H} \beta)$, and the extinction coefficient $c(\mathrm{H} \beta)$ for the integrated galaxy are tabulated in Table 3. In Table 4 we list the same quantities for the three SF regions in the BCD. We note that the mean extinction $c(\mathrm{H} \beta) \simeq 0.20$ we derive over HS $2236+1344$ is in good agreement with the value of 0.157 derived by Thuan \& Izotov (2005). Determinations for individual regions differ, however, from the values of 0.49 and 0.27 obtained by Izotov \& Thuan (2007) from the $\mathrm{H} \gamma / \mathrm{H} \beta$ ratio for their regions 1 and 2, respectively.

\subsection{Velocity field}

We have obtained the spatial distribution of radial velocities $v_{r}$ in the ISM by fitting a single Gaussian to the $\mathrm{H} \alpha$ emission line profiles. The velocity field of HS 2236+1344 is displayed in Fig. 4 . The $v_{r}$ displays a variation of $\sim 80 \mathrm{~km} \mathrm{~s}^{-1}$ over the FoV, while in the central part of the BCD it varies by about $45 \mathrm{~km} \mathrm{~s}^{-1}$. Additionally, the velocity field, in our FoV, close to the galaxy's center shows some rotation, with its upper part (northwest; region 1) being redshifted and its lower part (southeast; regions 2 and 3) blueshifted with respect to the systemic galaxy velocity. The relatively small measured variation in $v_{r}$ is in the range of 

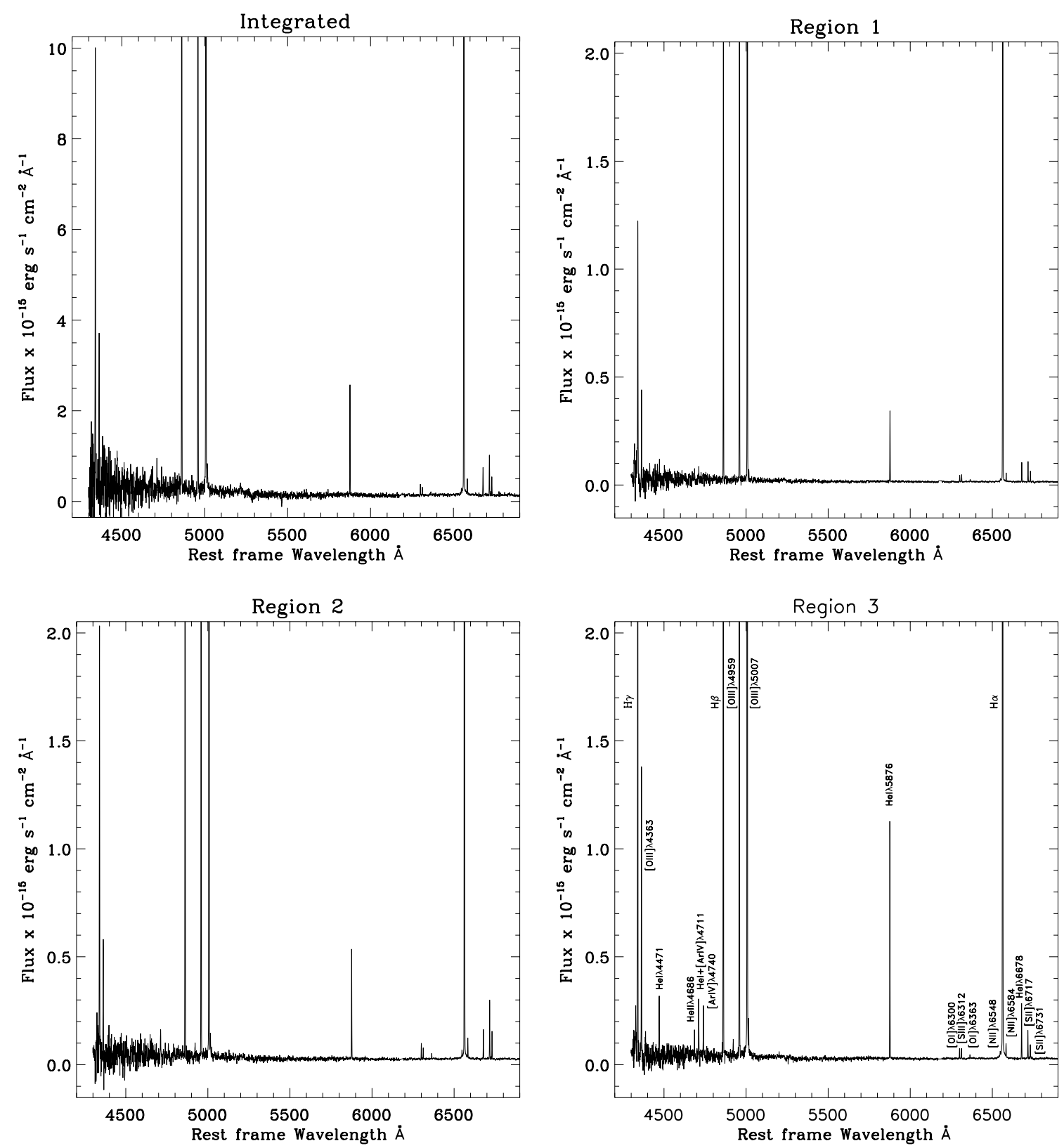

Fig. 2. Integrated Gemini GMOS spectrum of HS 2236+1344, and spectra extracted from regions 1-3 over the rest frame spectral range from $\sim 4300 \AA$ to $6900 \AA$. Note the presence of a broad low-intensity emission component in $\mathrm{H} \alpha$ (possibly also in $\mathrm{H} \beta$ ) and in the forbidden $[\mathrm{O}$ III $] \lambda 5007$ line.

values determined for other BCDs (e.g., van Zee et al. 2001; Cairós et al. 2012) yet significantly larger than for the intrinsically faint BCD UM 408 (Lagos et al. 2009).

Moiseev et al. (2010) studied the $\mathrm{H} \alpha$ velocity field of HS 2236+1344 using the multi-mode Fabry-Perot instrument SCORPIO. They concluded that, most likely, this galaxy is a strongly interacting pair of dwarf galaxies of comparable mass, possibly undergoing final stages of merging. Additionally, they argue that the observed kinematics can not be explained by giant ionized shells in a single rotating disc and, to the contrary, there is evidence for two independently rotating discs. Our $\mathrm{H} \alpha$ velocity field is similar to the one these authors determine, yet our data show no clear evidence for two kinematically distinct gas disks, or kinematical perturbations indicative of a strongly interacting system.

We checked for the presence of a broad low-intensity component in the $\mathrm{H} \alpha$ emission line profiles (Fig. 5) using the integrated spectrum of the galaxy, as well as that extracted from regions $1-3$. For this, we have followed a similar procedure as that described in Lagos et al. (2012): We used the pan ${ }^{2}$ routine (Peak ANalysis) in idl to fit two components to these profiles. Note that in different regions of the galaxy the $\mathrm{H} \alpha$ emission line profile is relatively symmetric and well represented by a single Gaussian. Although we use, in our study, the medium resolution grating R600, a detailed inspection of the base of the $\mathrm{H} \alpha$ emission line profile (and other emission lines, such as [O III] 15007) in regions 2 and 3 reveals a broad component at low intensity levels similar to the ones observed in other BCD galaxies (e.g., Izotov et al. 1996; Pustilnik et al. 2004; Izotov \& Thuan 2009). We fit the narrow component of the $\mathrm{H} \alpha$, [N II] $\lambda 6548$ and [N II] $\lambda 6584$ by a single Gaussians (see Fig. 5). Then, simultaneously, we fit the broad component by a single Gaussian obtaining

2 http://www.ncnr.nist.gov/staff/dimeo/panweb/pan.html 

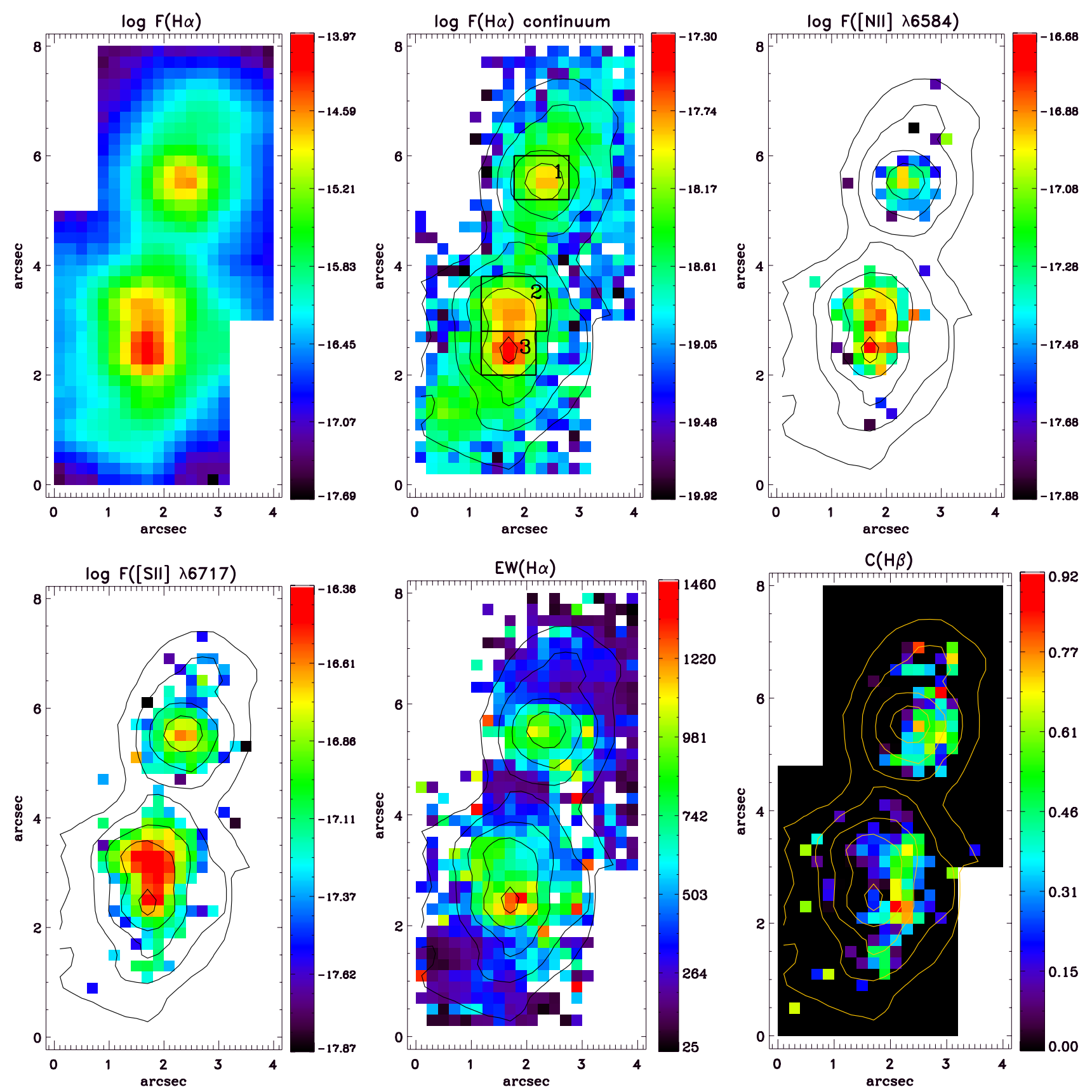

Fig. 3. Logarithmic emission line map of the $\mathrm{H} \alpha$ (top-left), $\mathrm{H} \alpha$ continuum (top-middle), [N II] 16584 (top-right), [S II] $\lambda 6717$ (bottom-left), EW(H $\alpha$ ) (bottom-middle) and extinction $c(\mathrm{H} \beta)$ (bottom-right). Logarithmic emission-line fluxes in units of erg cm $\mathrm{cm}^{-2} \mathrm{~s}^{-1}$, the logarithmic continuum flux density is in erg cm $\mathrm{cm}^{-2} \mathrm{~s}^{-1} \AA^{-1}$ and equivalent widths in $\AA$. Overlaid are the $\mathrm{H} \alpha$ flux contours. In the top-middle (H $\alpha$ continuum) panel we indicate the different apertures used in this study for regions $1-3$, respectively.

a FWHM of $\gtrsim 100 \AA$. A detailed analysis of this component is beyond the scope of this paper but its presence, in the inner part of region 3, appears to be consistent with stellar winds due by massive stars, e.g., from Luminous Blue Variables (LBV).

\subsection{The He Il $\lambda 4686$ emission line}

In Fig. 6 we show the integrated spectrum of region 3 in the wavelength range from $4600 \AA$ to $4900 \AA$, which reveals the presence of a narrow He II $\lambda 4686$ emission. Figure 7, encompassing regions 2 and 3, allows a study of the spatial distribution of that line. Squares represent the area on the sky that is covered by individual spaxels and are overlaid with the respective observed spectrum in the wavelength range $4600-4800 \AA$.

It is apparent from Fig. 7 that the high-ionization He II $\lambda 4686$ emission is not extended such as in the compact $\mathrm{H}$ II/BCD galaxies Tol 2146-391 (Lagos et al. 2012) and Mrk 178 (Kehrig et al. 2013). On the contrary, we find that most of the He II $\lambda 4686$ emission in HS $2236+1344$ is confined within region 3 (see Fig. 7), near the peak of $\mathrm{H} \alpha$ emission. This is presumably due to the low $\mathrm{S} / \mathrm{N}$ in the blue part of our GMOS spectra, which likely prevents detection of extended low-surface brightness in $\mathrm{He}$ II emission that may be present. This also suggested by the fact that previous high-S/N long slit spectroscopy (Izotov \& Thuan 2007) has revealed He II emission in region \#1 as well. In any case, the spatial association of He II $\lambda 4686$ with region \#3 


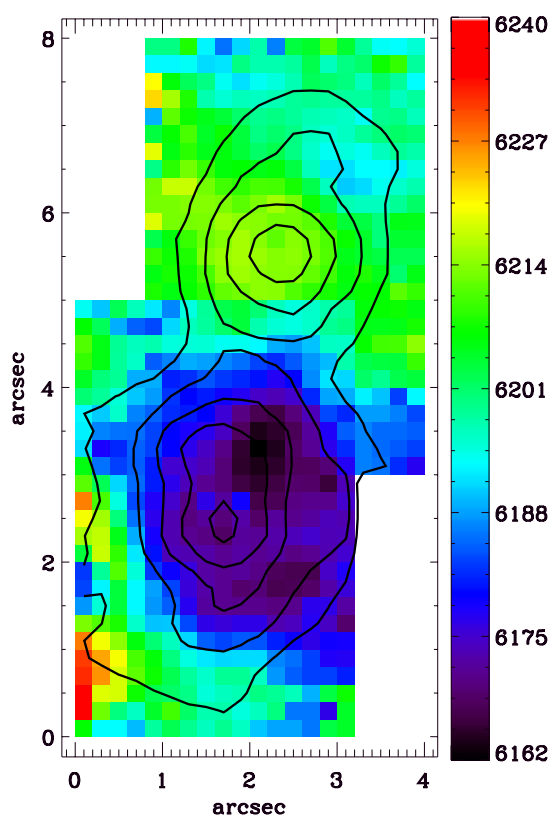

Fig. 4. Recessional velocity of the $\mathrm{H} \alpha$ emission line in units of $\mathrm{km} \mathrm{s}^{-1}$. Contours display the $\mathrm{H} \alpha$ morphology of HS $2236+1344$.

suggests that its excitation is due to the ongoing burst. We note that the intensity $I(\mathrm{He}$ II $\lambda 4686) / I(\mathrm{H} \beta)=0.0122 \pm 0.0029$ in region 3 agrees within the errors with $I(\mathrm{He}$ II $\lambda 4686) / I(\mathrm{H} \beta)=$ $0.0105 \pm 0.0008$ and $0.0112 \pm 0.0013$ obtained by Izotov \& Thuan (2007) for their regions 1 and 2, respectively.

One of the most likely explanations ${ }^{3}$ for the high ionization emission lines in BCD galaxies is the presence of fast radiative shocks (Dopita \& Sutherland 1996) in the ISM of these galaxies (e.g., Thuan \& Izotov 2005; Izotov et al. 2006a; Lagos et al. 2012). As noted above, we do not detect the He II $\lambda 4686$ emission line at the arm-like structures in HS $2236+1344$ or within any other extended morphological feature. On the other hand, at ages of 3-6 Myr the WR phase is still going on (Leitherer et al. 1999), so we expect a significant amount of these stars in the young regions (with ages of $~ 3 \mathrm{Myr}$; see Sect. 3.7 for more details) found in this galaxy. But, we did not detect clear WR features at $\lambda 4686 \AA$ neither in the integrated spectrum of the galaxy nor in the different regions resolved in HS 2236+1344. Although, in the case of WR stars the He II $\lambda 4686$ emission line and the blue WR bump are mainly linked to WN stars, the non-detection of this stellar emission suggests the existence of WR stars in an early evolutionary stage (e.g., LBV stars). In fact, a large fraction of galaxies with oxygen abundances lower than $12+\log (\mathrm{O} / \mathrm{H})=8.2$ do not show WR features (Guseva et al. 2000; Brinchmann et al. 2008; Shirazi \& Brinchmann 2012). It is also known that in several $\mathrm{H}$ II/BCDs (e.g., II Zw 70 and Mrk 178; Kehrig et al. 2008, 2013) He II $\lambda 4686$ emission does not strictly coincide with the location of the WR bumps. In this sense, He II emission without associated WR features does not completely rule out a contribution of WR stars to the He II ionization, even though other excitation sources are likely dominating.

In summary, various ionization sources may appear consistent with the observed morphology of the He II $\lambda 4686$ emission in HS $2236+1344$, whereas from the present data this seems to be an unresolved issue.

\footnotetext{
3 See, e.g., Thuan \& Izotov (2005) and references therein for other candidate sources for He II $\lambda 4686$ ionization.
}
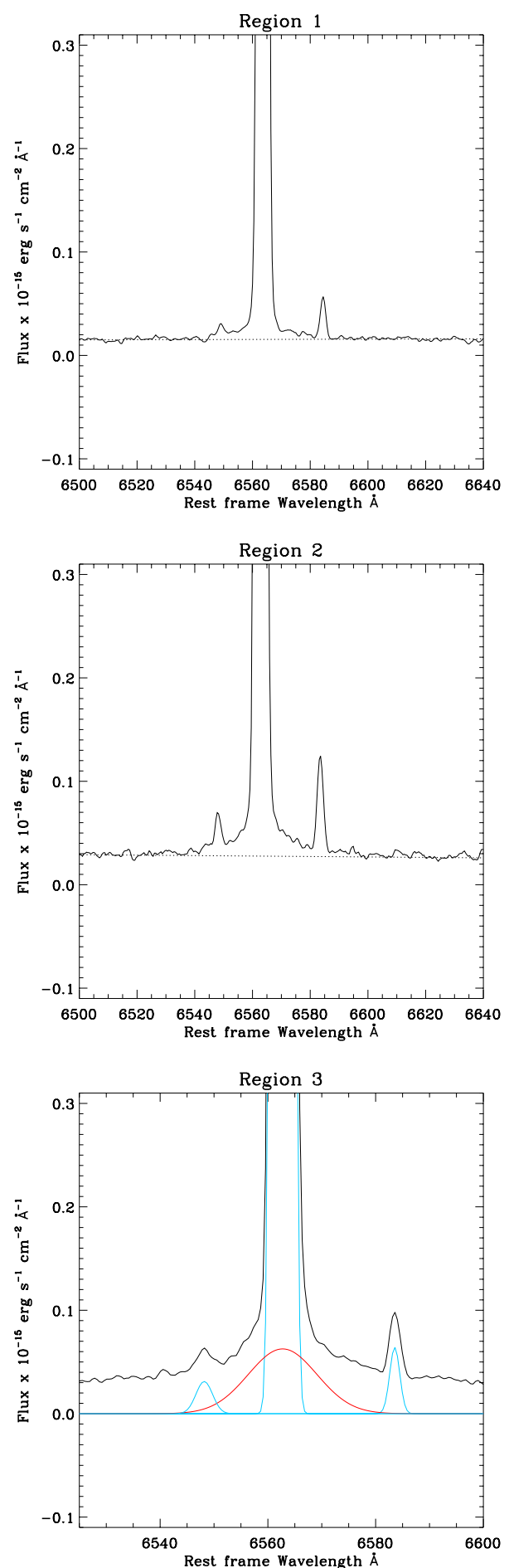

Fig. 5. $\mathrm{H} \alpha$ emission line profiles of regions 1-3, respectively. Note the presence of broad emission (red line) in region 3.

\subsection{Emission line ratios}

Using the information derived in the previous sections it is possible to attempt distinguishing among different ionization mechanisms using BPT (Baldwin et al. 1981) diagrams. To do this, we adopt the following emission line ratios: [O III] $\lambda 5007 / \mathrm{H} \beta$, [N II] $\lambda 6584 / \mathrm{H} \alpha$, [S II] $\lambda \lambda 6717,6731 / \mathrm{H} \alpha$ and [O I] $\lambda 6300 / \mathrm{H} \alpha$. The [O III] $\lambda 5007 / \mathrm{H} \beta$ emission line ratio is an excitation indicator and provides information about the available fraction of hard ionizing photons in an H II region. On the other hand, [N II] $\lambda 6584$, [S II] $\lambda \lambda 6717,6731$ and [O I] $\lambda 6300$ are 


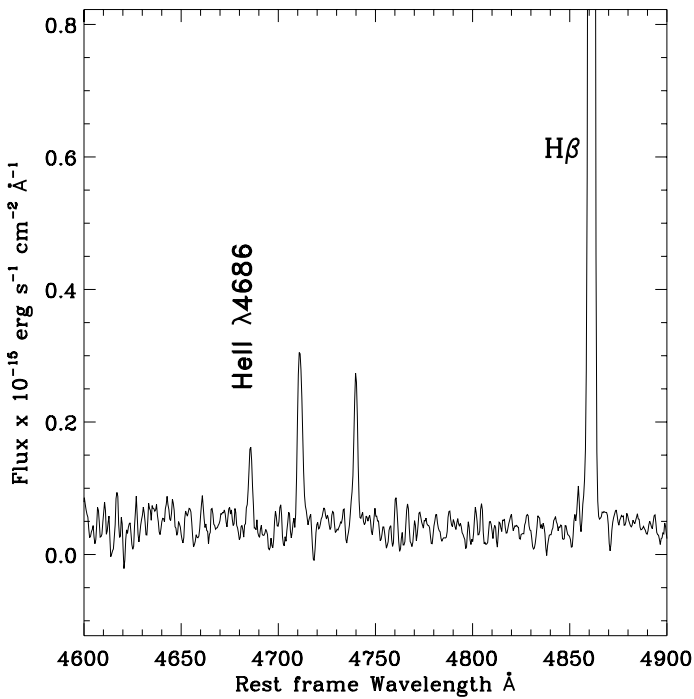

Fig. 6. Integrated spectrum of HS $2236+1344$ between $4600 \AA$ to $4900 \AA$ with the He II $\lambda 4686$ and $\mathrm{H} \beta$ emission lines labeled.

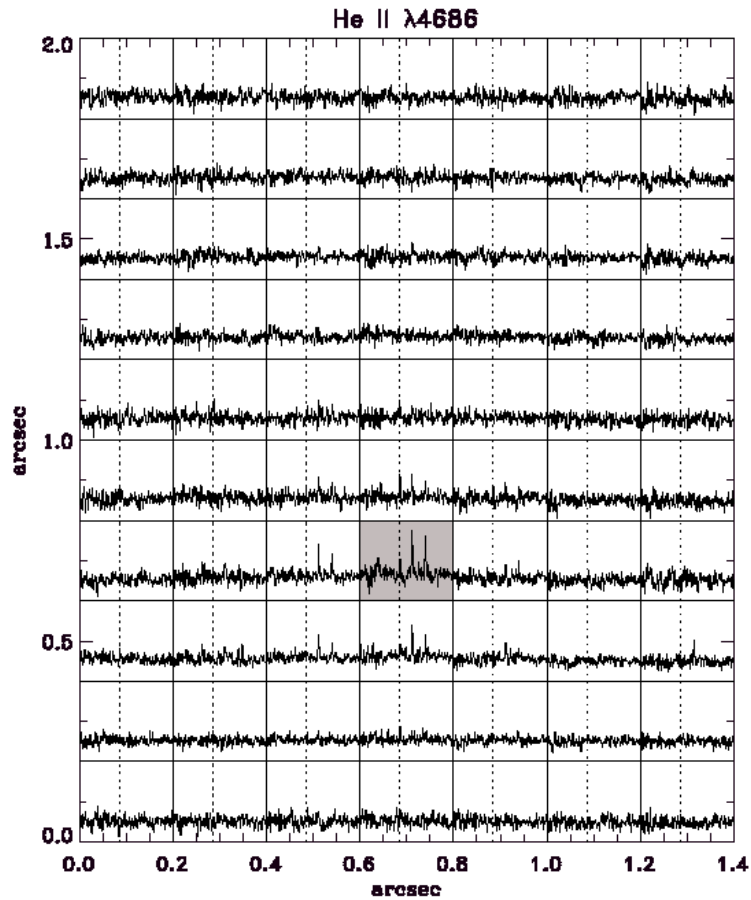

Fig. 7. Spectral region between $4600 \AA$ to $4800 \AA$ that includes the He II $\lambda 4686$ emission line in a rectangular area of the galaxy that contains regions 2 and 3. Each square represents the area covered by an individual spaxel in that region. The He II emission line in each spectrum is indicated by the dotted line. The dark square corresponds to the $\mathrm{H} \alpha$ peak.

low-ionization emission lines, usually weak in $\mathrm{H}$ II regions. So, the emission line ratios [N II] 6584/H $\alpha$, [S II] $\lambda \lambda 6717,6731 / \mathrm{H} \alpha$ and [O I] $\lambda 6300 / \mathrm{H} \alpha$ can effectively disentangle between photoionization under physical conditions that are typical for $\mathrm{H}$ II regions and other excitation mechanisms (e.g., AGN or shocks).

In Fig. 8 we show the emission line ratio maps [O III] $\lambda 5007 / \mathrm{H} \beta$, [N II] $\lambda 6584 / \mathrm{H} \alpha,[\mathrm{S} \mathrm{II}] \lambda \lambda 6717,6731 / \mathrm{H} \alpha$ and [O I] $\lambda 6300 / \mathrm{H} \alpha$, respectively. In this figure, the ionization structure in the central part of regions 1, and 3 of HS 2236+1344 is rather uniform for all emission line ratios, while the emission line ratio $[\mathrm{O} \mathrm{III}] \lambda 5007 / \mathrm{H} \beta$ decreases slightly, in region 2 ,
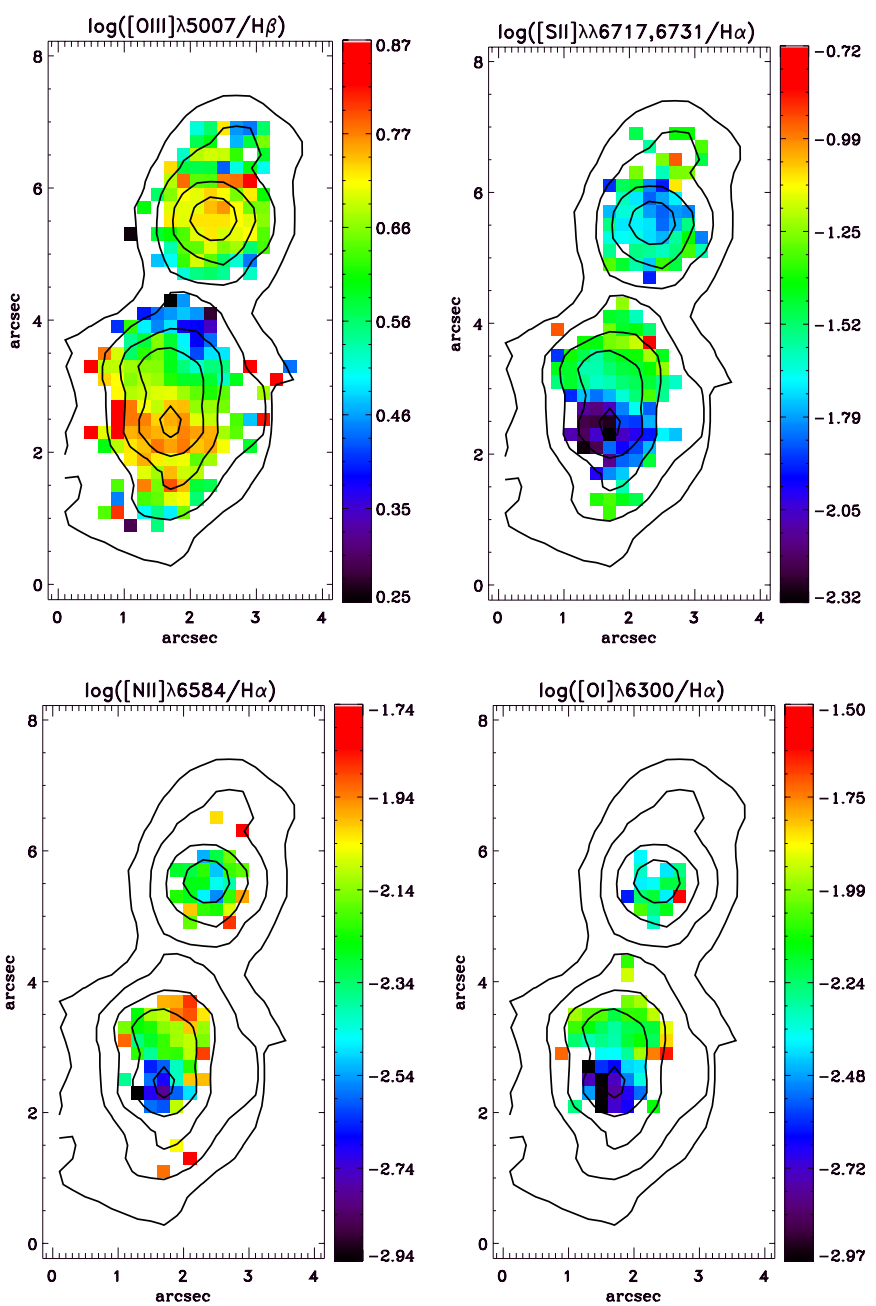

Fig. 8. Emission line ratios: $\log [\mathrm{O} \mathrm{III}] \lambda 5007 / \mathrm{H} \beta, \log [\mathrm{S} \mathrm{II}] \lambda \lambda 6717$, $6731 / \mathrm{H} \alpha, \log [\mathrm{N} \mathrm{II}] \lambda 6584 / \mathrm{H} \alpha$ and $[\mathrm{O} \mathrm{I}] \lambda 6300 / \mathrm{H} \alpha$. Overlaid are the $\mathrm{H} \alpha$ flux contours.

with distance. The opposite is observed for the [S II] $\lambda \lambda 6717$, $6731 / \mathrm{H} \alpha,[\mathrm{N}$ II] $\lambda 6584 / \mathrm{H} \alpha$ and [O I] $\lambda 6300 / \mathrm{H} \alpha$ ratios.

In Fig. 9 we show the BPT diagram comparing [O III] $\lambda 5007 / \mathrm{H} \beta$ versus [N II] $\lambda 6584 / \mathrm{H} \alpha$. It can be seen from Fig. 9 that the data points of HS $2236+1344$ occupy the left region of the BPT diagram, showing smaller [N II] $\lambda 6584 / \mathrm{H} \alpha$ ratios, while the $[\mathrm{O} \mathrm{III}] \lambda 5007 / \mathrm{H} \beta$ ratio increases. Therefore, the data points of HS $2236+1344$ occupy the same region of the diagram, the left part of the BPT diagram, than other XMP galaxies found in the literature (Izotov et al. 2012).

Finally, we note that our integrated [O III] $\lambda 5007 / \mathrm{H} \beta$, [S II] $\lambda \lambda 6717,6731 / \mathrm{H} \alpha, \quad[\mathrm{N} \mathrm{II}] \lambda 6584 / \mathrm{H} \alpha$ and [O I] $\lambda 6300 / \mathrm{H} \alpha$ values for HS $2236+1344$ (Table 5) are consistent with the ones derived by Thuan \& Izotov (2005) of 0.68 , $-1.65,-2.21$ and -2.39 , respectively.

\subsection{Chemical abundances}

In order to determine the chemical abundance pattern in the ISM of HS 2236+1344, we used the five level atomic model FIVEL (De Robertis et al. 1987), implemented in the IRAF STSDAS package nebular. We first calculated the electron temperature $T_{\mathrm{e}}(\mathrm{O}$ III $)$ from the ratio [O III] $\lambda \lambda 4959,5007 /[\mathrm{O}$ III] $\lambda 4363$ and the electron density from the ratio [S II] $\lambda 6717 /[\mathrm{S}$ II] $\lambda 6731$. The latter is typically $>1$, which indicates a low electron 


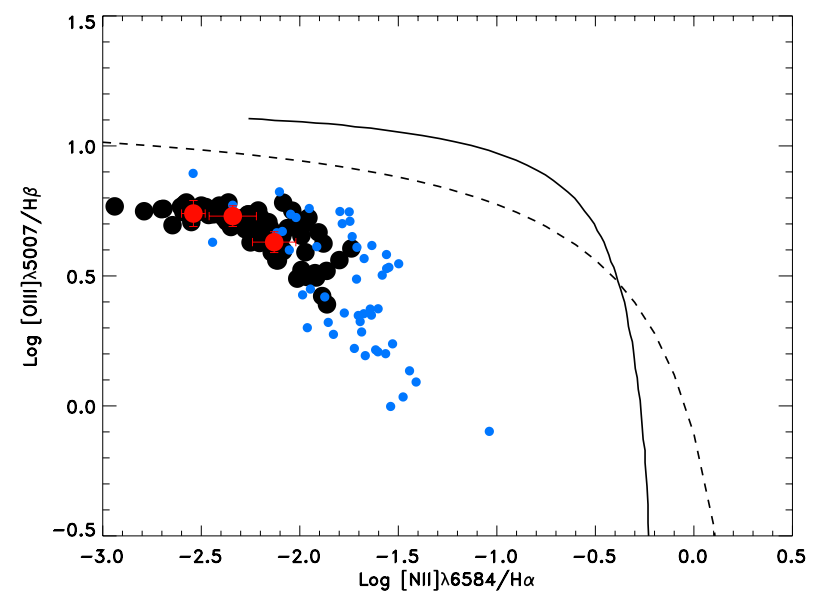

Fig. 9. $\log [\mathrm{O} \mathrm{III}] \lambda 5007 / \mathrm{H} \beta$ versus $\log [\mathrm{N} \mathrm{II}] \lambda 6584 / \mathrm{H} \alpha$ BPT diagram. The solid curves show the empirical borders between photoionization by massive stars and other excitation mechanisms (Osterbrock \& Ferland 2006), while the dotted lines show the demarcation proposed by Kewley et al. (2001). Black dots correspond to individual spaxels in Fig. 8. The values for region $1-3$ of HS $2236+1344$ are indicated in the diagram by red points. Blue data points show several low metallicity $(12+\log (\mathrm{O} / \mathrm{H}) \lesssim 7.6) \mathrm{SF}$ regions from Izotov et al. (2012).

density $n_{\mathrm{e}}$ (Osterbrock \& Ferland 2006). Large [S II] $\lambda 6717 /$ [S II] $\lambda 6731$ ratios were already observed in other studies (e.g., Lagos et al. 2009; López-Hernández et al. 2013; Krabbe et al. 2014). These nonphysical values could be partly due to associated with uncertainties in the measurements of these emission lines, due to the placement of the continuum or, more likely, because the spectrum was not corrected for night sky absorption lines. Therefore, intensities of [S II] $\lambda 6717$ and [S II] $\lambda 6731$ (Tables 3 and 4) lines are, likely, affected by the night sky absorption. In any case, we compare our results with the SDSS spectrum of the same galaxy obtaining [S II] $\lambda 6717 /[$ S II] $\lambda 6731 \sim 1.6$, which is about $20 \%$ greater than the value obtained by Thuan \& Izotov (2005) of $1.31\left(\sim 110 \mathrm{~cm}^{-3}\right)$. Therefore, it is reasonable to assume a value of $100 \mathrm{~cm}^{-3}$ for all apertures in our calculations.

The oxygen and nitrogen abundances were calculated as

$\frac{\mathrm{O}}{\mathrm{H}}=\frac{\mathrm{O}^{+}}{\mathrm{H}^{+}}+\frac{\mathrm{O}^{+2}}{\mathrm{H}^{+}}$

and

$\frac{\mathrm{N}}{\mathrm{H}}=\operatorname{ICF}(\mathrm{N}) \frac{\mathrm{N}^{+}}{\mathrm{H}^{+}}$,

with the ionic abundances $\mathrm{O}^{+}, \mathrm{O}^{+2}$ and $\mathrm{N}^{+}$obtained from the nebular output file. Nitrogen abundances were calculated using an ionization correction factor $(\mathrm{ICF}) \operatorname{ICF}(\mathrm{N})=\left(\mathrm{O}^{+}+\mathrm{O}^{+2}\right) / \mathrm{O}^{+}$. We assumed that the $\mathrm{T}_{\mathrm{e}}(\mathrm{O}$ II $)$ temperature is given by $\mathrm{T}_{\mathrm{e}}(\mathrm{O}$ II $)=$ $2 /\left(\mathrm{T}_{\mathrm{e}}^{-1}(\mathrm{O}\right.$ III $\left.)+0.8\right)$ (Pagel et al. 1992) and $\mathrm{T}_{\mathrm{e}}(\mathrm{O} \mathrm{II})=\mathrm{T}_{\mathrm{e}}(\mathrm{N}$ II $)$. Since the [O II] $\lambda 3727$ doublet is not within the spectral range of our data, we have to rely on published fluxes. We therefore adopted ratios of $F(\mathrm{O}$ II $\lambda 3727) / F(\mathrm{H} \beta)=54.67$ (Thuan \& Izotov 2005) for the integrated spectrum, $40.33 \pm 0.69$ for region 1 and $25.39 \pm 0.41$ for regions 2 and 3 (Izotov \& Thuan 2007), respectively. The fluxes are relative to $F(\mathrm{H} \beta)=100$. We checked our results with an alternative method for deriving abundances in which a constant $F(\mathrm{O}$ II $\lambda 3727) / F(\mathrm{H} \beta)$ value is assumed (Thuan \& Izotov 2005). We found that values derived using a constant $F(\mathrm{O}$ II $\lambda 3727) / F(\mathrm{H} \beta)$ ratio yield oxygen abundances $\sim 0.03$ dex higher and nitrogen abundances $\sim 0.23$ dex
Table 3. Observed and extinction corrected emission line fluxes for the integrated spectrum of the galaxy.

\begin{tabular}{|c|c|c|}
\hline & \multicolumn{2}{|c|}{ Integrated } \\
\hline & $F(\lambda) / F(\mathrm{H} \beta)$ & $I(\lambda) / I(\mathrm{H} \beta)$ \\
\hline Н $\gamma \lambda 4340$ & $46.12 \pm 3.88$ & $49.21 \pm 7.31$ \\
\hline [O III] $] \lambda 4363$ & $14.75 \pm 0.95$ & $15.69 \pm 1.36$ \\
\hline Не г $\lambda 4471$ & $>4.30 \pm 0.39$ & $>4.51 \pm 0.59$ \\
\hline Не II $\lambda 4686$ & $>1.20 \pm 0.20$ & $>1.22 \pm 0.29$ \\
\hline $\mathrm{He} \mathrm{I}+[\mathrm{Ar} \mathrm{V}] \lambda 4711$ & $>3.80 \pm 0.53$ & $>3.87 \pm 0.76$ \\
\hline$[\mathrm{ArV}] \lambda 4740$ & $>2.70 \pm 0.14$ & $>2.74 \pm 0.20$ \\
\hline $\mathrm{H} \beta \lambda 4861$ & $100.00 \pm 2.71$ & $100.00 \pm 3.83$ \\
\hline [O III] $\lambda 4959$ & $169.96 \pm 5.02$ & $168.61 \pm 7.05$ \\
\hline [O III] $] \lambda 5007$ & $507.75 \pm 12.70$ & $499.87 \pm 17.68$ \\
\hline Не I $\lambda 5876$ & $11.92 \pm 0.68$ & $10.96 \pm 0.88$ \\
\hline [O I] $] \lambda 6300$ & $1.03 \pm 0.20$ & $0.92 \pm 0.25$ \\
\hline [S III] $] \lambda 6312$ & $1.10 \pm 0.12$ & $0.99 \pm 0.14$ \\
\hline [O I] $\lambda 6363$ & $0.32 \pm 0.03$ & $0.29 \pm 0.04$ \\
\hline [N II] $] 26548$ & $0.61 \pm 0.10$ & $0.54 \pm 0.16$ \\
\hline Н $\alpha \lambda 6563$ & $317.83 \pm 6.25$ & $280.95 \pm 7.81$ \\
\hline$[\mathrm{N}$ II $] \lambda 6584$ & $2.05 \pm 0.14$ & $1.81 \pm 0.17$ \\
\hline Не I $\lambda 6678$ & $3.43 \pm 0.45$ & $3.01 \pm 0.56$ \\
\hline [S II] $] \lambda 6717$ & $4.94 \pm 0.30$ & $4.33 \pm 0.37$ \\
\hline$[\mathrm{S}$ II $] \lambda 6731$ & $2.13 \pm 0.38$ & $1.86 \pm 0.47$ \\
\hline$F(\mathrm{H} \beta)^{a}$ & $5.16 \pm 0.07$ & \\
\hline $\mathrm{EW}(\mathrm{H} \alpha)^{b}$ & 834 & \\
\hline $\mathrm{EW}(\mathrm{H} \beta)^{b}$ & 157 & \\
\hline$c(\mathrm{H} \beta)$ & 0.18 & \\
\hline
\end{tabular}

Notes. The fluxes are relative to $F(\mathrm{H} \beta)=100 .{ }^{(a)}$ In units of $\times 10^{-14} \mathrm{erg} \mathrm{cm}^{-2} \mathrm{~s}^{-1} ;{ }^{(b)}$ in units of $\AA$.

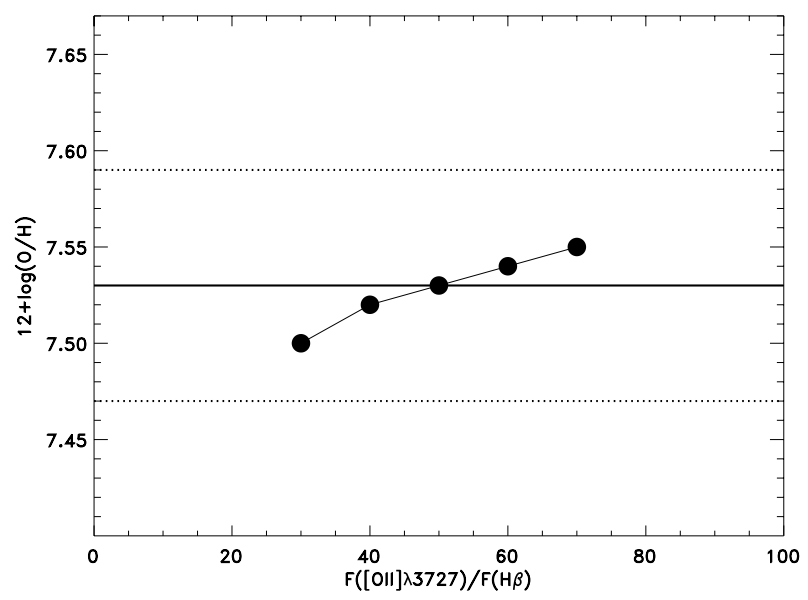

Fig. 10. Variation of the $12+\log (\mathrm{O} / \mathrm{H})$ abundance in the integrated galaxy (black data points) as a function of the adopted $F([\mathrm{OII}] \lambda 3727) / F(\mathrm{H} \beta)$ ratio. The fluxes are relative to $F(\mathrm{H} \beta)=100$. The continuum lines corresponds to the integrated values of 7.53 and the dotted lines show the uncertainties associated with these values. More details are in the text.

lower than the ones obtained using the values of Izotov \& Thuan (2007). In Fig. 10 we show the variation of the $12+\log (\mathrm{O} / \mathrm{H})$ abundance in the integrated galaxy as a function of the adopted $F([\mathrm{O}$ II $] \lambda 3727) / F(\mathrm{H} \beta)$ ratio. From this figure we can see that very small changes were found by adopting the values by Thuan \& Izotov (2005) and Izotov \& Thuan (2007), since the oxygen abundance do not vary strongly with $F(\mathrm{O}$ II $\lambda 3727) / F(\mathrm{H} \beta)$ within the errors. So, the adoption of [O II] $\lambda 3727$ fluxes from the literature introduces only minor uncertainties in the analysis of $12+\log (\mathrm{O} / \mathrm{H})$, while the N/O abundance ratio is highly uncertain. 
Table 4. Observed and extinction corrected emission lines for the regions 1-3.

\begin{tabular}{|c|c|c|c|c|c|c|}
\hline & \multicolumn{2}{|c|}{ Region 1} & \multicolumn{2}{|c|}{ Region 2} & \multicolumn{2}{|c|}{ Region 3} \\
\hline & $F(\lambda) / F(\mathrm{H} \beta)$ & $I(\lambda) / I(\mathrm{H} \beta)$ & $F(\lambda) / F(\mathrm{H} \beta)$ & $I(\lambda) / I(\mathrm{H} \beta)$ & $F(\lambda) / F(\mathrm{H} \beta)$ & $I(\lambda) / I(\mathrm{H} \beta)$ \\
\hline $\mathrm{H} \gamma \lambda 4340$ & $45.15 \pm 2.27$ & $48.70 \pm 3.46$ & $46.70 \pm 2.93$ & $50.01 \pm 4.44$ & $47.80 \pm 2.89$ & $49.56 \pm 4.24$ \\
\hline [O III] $\lambda 4363$ & $16.29 \pm 0.86$ & $17.51 \pm 1.31$ & $13.76 \pm 0.89$ & $14.69 \pm 1.34$ & $17.43 \pm 0.64$ & $18.04 \pm 0.94$ \\
\hline He I $\lambda 4471$ & $\ldots$ & $\ldots$ & $\ldots$ & $\ldots$ & $4.30 \pm 0.39$ & $4.42 \pm 0.57$ \\
\hline HeII $\lambda 4686$ & $\ldots$ & $\ldots$ & $\ldots$ & $\ldots$ & $1.20 \pm 0.20$ & $1.22 \pm 0.29$ \\
\hline $\mathrm{He} \mathrm{I}+[\mathrm{Ar} \mathrm{V}] \lambda 4711$ & $\ldots$ & $\ldots$ & $\ldots$ & $\ldots$ & $3.80 \pm 0.53$ & $3.84 \pm 0.76$ \\
\hline$[\mathrm{ArV} \mathrm{V}] \lambda 4740$ & $\ldots$ & $\ldots$ & $\ldots$ & $\ldots$ & $2.70 \pm 0.14$ & $2.72 \pm 0.20$ \\
\hline $\mathrm{H} \beta \lambda 4861$ & $100.00 \pm 1.22$ & $100.00 \pm 1.73$ & $100.00 \pm 3.69$ & $100.00 \pm 5.22$ & $100.00 \pm 3.14$ & $100.00 \pm 4.44$ \\
\hline [O III] $] \lambda 4959$ & $179.60 \pm 7.18$ & $177.38 \pm 10.03$ & $143.12 \pm 3.54$ & $141.52 \pm 4.95$ & $186.38 \pm 6.07$ & $185.28 \pm 8.53$ \\
\hline [O III] $] \lambda 5007$ & $547.94 \pm 9.42$ & $538.03 \pm 13.08$ & $431.19 \pm 9.75$ & $424.13 \pm 13.56$ & $554.97 \pm 19.19$ & $550.17 \pm 26.90$ \\
\hline He I $\lambda 5876$ & $116.59 \pm 1.32$ & $105.68 \pm 1.69$ & $10.92 \pm 0.38$ & $9.99 \pm 0.49$ & $12.98 \pm 0.67$ & $12.39 \pm 0.90$ \\
\hline [O I] $\lambda 6300$ & $1.10 \pm 0.13$ & $0.97 \pm 0.16$ & $1.58 \pm 0.12$ & $1.41 \pm 0.15$ & $0.53 \pm 0.02$ & $0.50 \pm 0.03$ \\
\hline [S III] $] \lambda 6312$ & $1.22 \pm 0.16$ & $1.07 \pm 0.20$ & $1.05 \pm 0.12$ & $0.93 \pm 0.15$ & $0.54 \pm 0.02$ & $0.51 \pm 0.03$ \\
\hline [O I] $\lambda 6363$ & $0.41 \pm 0.06$ & $0.36 \pm 0.07$ & $0.52 \pm 0.05$ & $0.46 \pm 0.06$ & $0.17 \pm 0.03$ & $0.16 \pm 0.04$ \\
\hline$[\mathrm{N}$ II] $] \lambda 6548$ & $0.40 \pm 0.10$ & $0.35 \pm 0.14$ & $0.71 \pm 0.09$ & $0.62 \pm 0.19$ & $0.26 \pm 0.06$ & $0.24 \pm 0.08$ \\
\hline $\mathrm{H} \alpha \lambda 6563$ & $324.20 \pm 3.49$ & $280.75 \pm 4.27$ & $321.10 \pm 6.81$ & $281.90 \pm 8.45$ & $298.95 \pm 5.22$ & $279.15 \pm 6.89$ \\
\hline [N II] $] 26584$ & $1.48 \pm 0.11$ & $1.28 \pm 0.13$ & $2.39 \pm 0.13$ & $2.10 \pm 0.16$ & $0.87 \pm 0.02$ & $0.81 \pm 0.03$ \\
\hline HeI $\lambda 6678$ & $3.32 \pm 0.34$ & $2.85 \pm 0.41$ & $3.15 \pm 0.25$ & $2.75 \pm 0.31$ & $3.47 \pm 0.15$ & $3.23 \pm 0.20$ \\
\hline [S II] $] \lambda 6717$ & $3.88 \pm 0.21$ & $3.33 \pm 0.25$ & $6.29 \pm 0.35$ & $5.47 \pm 0.43$ & $1.64 \pm 0.07$ & $1.52 \pm 0.09$ \\
\hline [S II] $] \lambda 6731$ & $1.67 \pm 0.28$ & $1.43 \pm 0.34$ & $2.92 \pm 0.25$ & $2.54 \pm 0.31$ & $0.70 \pm 0.07$ & $0.65 \pm 0.09$ \\
\hline$F(\mathrm{H} \beta)^{a}$ & $0.66 \pm 0.01$ & & $1.09 \pm 0.02$ & & $1.91 \pm 0.03$ & \\
\hline $\mathrm{EW}(\mathrm{H} \alpha)^{b}$ & 970 & & 829 & & 1311 & \\
\hline $\mathrm{EW}(\mathrm{H} \beta)^{b}$ & 272 & & 233 & & 300 & \\
\hline$c(\mathrm{H} \beta)$ & 0.21 & & 0.19 & & 0.10 & \\
\hline
\end{tabular}

Notes. The fluxes are relative to $F(\mathrm{H} \beta)=100 .{ }^{(a)}$ In units of $\times 10^{-14} \mathrm{erg} \mathrm{cm}^{-2} \mathrm{~s}^{-1}{ }^{(b)}$ in units of $\AA$.

Table 5. Ionic abundances and integrated properties of HS 2236+1344.

\begin{tabular}{lcccc}
\hline \hline & Integrated & Region 1 & Region 2 & Region 3 \\
\hline $\mathrm{Te}(\mathrm{O}$ III $) \mathrm{K}$ & $19442 \pm 443$ & $19925 \pm 635$ & $20665 \pm 584$ & $19951 \pm 333$ \\
$\mathrm{Ne}(\mathrm{S} \mathrm{II}) \mathrm{cm}^{--3}$ & $\sim 100$ & $\sim 100$ & $\sim 100$ & $\sim 100$ \\
$\mathrm{O}^{+} / \mathrm{H}^{+} \times 10^{5}$ & $0.51 \pm 0.02$ & $0.37 \pm 0.02$ & $0.22 \pm 0.01$ & $0.21 \pm 0.01$ \\
$\mathrm{O}^{++} / \mathrm{H}^{+} \times 10^{5}$ & $2.92 \pm 0.20$ & $2.97 \pm 0.28$ & $2.18 \pm 0.17$ & $3.05 \pm 0.15$ \\
$\mathrm{O} / \mathrm{H} \times 10^{5}$ & $3.43 \pm 0.22$ & $3.35 \pm 0.30$ & $2.40 \pm 0.19$ & $3.26 \pm 0.15$ \\
$12+\log (\mathrm{O} / \mathrm{H})$ & $7.53 \pm 0.06$ & $7.52 \pm 0.09$ & $7.38 \pm 0.08$ & $7.51 \pm 0.05$ \\
$\mathrm{~N}^{+} / \mathrm{H}^{+} \times 10^{6}$ & $0.14 \pm 0.01$ & $0.09 \pm 0.01$ & $0.15 \pm 0.01$ & $0.06 \pm 0.01$ \\
$\mathrm{ICF}(\mathrm{N})$ & $6.77 \pm 0.69$ & $9.02 \pm 1.28$ & $10.90 \pm 1.34$ & $15.20 \pm 1.14$ \\
$\mathrm{~N} / \mathrm{H} \times 10^{6}$ & $0.93 \pm 0.12$ & $0.86 \pm 0.15$ & $1.65 \pm 0.26$ & $0.92 \pm 0.08$ \\
$12+\log (\mathrm{N} / \mathrm{H})$ & $5.97 \pm 0.13$ & $5.93 \pm 0.18$ & $6.22 \pm 0.15$ & $5.96 \pm 0.09$ \\
$\log (\mathrm{N} / \mathrm{O})$ & $-1.57 \pm 0.19$ & $-1.59 \pm 0.27$ & $-1.16 \pm 0.23$ & $-1.55 \pm 0.14$ \\
\hline $\mathrm{He} \mathrm{II} \lambda 4686 / \mathrm{H} \beta$ & $\cdots$ & $\cdots$ & $\cdots$ & $0.0122 \pm 0.0029$ \\
$\log ([\mathrm{O} \mathrm{III}] \lambda 5007 / \mathrm{H} \beta)$ & $0.70 \pm 0.03$ & $0.73 \pm 0.02$ & $0.63 \pm 0.03$ & $0.74 \pm 0.05$ \\
$\log ([\mathrm{N} \mathrm{II}] \lambda 6584 / \mathrm{H} \alpha)$ & $-2.19 \pm 0.12$ & $-2.34 \pm 0.12$ & $-2.13 \pm 0.11$ & $-2.54 \pm 0.06$ \\
$\log ([\mathrm{S} \mathrm{II}] \lambda \lambda 6717,6731 / \mathrm{H} \alpha)$ & $-1.66 \pm 0.16$ & $-1.77 \pm 0.14$ & $-1.55 \pm 0.12$ & $-2.11 \pm 0.12$ \\
$\log ([\mathrm{O} \mathrm{I}] \lambda 6300 / \mathrm{H} \alpha)$ & $-2.48 \pm 0.30$ & $-2.46 \pm 0.18$ & $-2.30 \pm 0.14$ & $-2.75 \pm 0.08$ \\
\hline
\end{tabular}

In Table 5 we show the electron density, electron temperature and abundances calculated for each one of the integrated apertures considered in this work. Figure 11 (left panel) shows the spatial distribution of the oxygen abundances in spaxels with detected [O III] $\lambda 4363$ emission, and in the right panel of the same figure we show the spatial distribution of $\log (\mathrm{N} / \mathrm{O})$ calculated using the oxygen and nitrogen maps in the spaxels with detected [N II] $\lambda 6584$ emission.

The oxygen abundance in the galaxy varies from 7.31 to 7.72 (see Fig. 11). The integrated and mean values are almost equal for regions 1 and 3, with values of $12+\log (\mathrm{O} / \mathrm{H})=7.52 \pm 0.09$ and $7.51 \pm 0.05$, respectively. While, in region 2 , we found an oxygen abundance of $12+\log (\mathrm{O} / \mathrm{H})=7.38 \pm 0.08$. The integrated abundance $12+\log (\mathrm{O} / \mathrm{H})=7.53 \pm 0.06$ determined here agrees, within the errors, with the value reported by Thuan \& Izotov (2005) of $7.473 \pm 0.012$. Our oxygen abundance determination for region 1-3 agrees, within the errors, with the ones found by Izotov \& Thuan (2007) with $12+\log (\mathrm{O} / \mathrm{H})=$ $7.450 \pm 0.012$ and $7.562 \pm 0.013$, for their regions 1 and 2 , respectively. With regard to the nitrogen abundance, we derive an integrated value of $12+\log (\mathrm{N} / \mathrm{H})=5.97 \pm 0.13$ which is consistent, within the errors, with the values in individual regions $(5.93 \pm 0.18,6.22 \pm 0.15$ and $5.96 \pm 0.09$, for regions $1-3$, respectively). The $\log (\mathrm{N} / \mathrm{O})$ ratio (Fig. 11) ranges from -1.85 to -1.39 with a mean value of $-1.60 \pm 0.16$ and $-1.68 \pm 0.10$ in regions 1 and 3 , respectively. Finally, we found an integrated value of $\log (\mathrm{N} / \mathrm{O})=-1.57 \pm 0.19$ and $\log (\mathrm{N} / \mathrm{O})=-1.59 \pm 0.27$ and $-1.55 \pm 0.14$ for regions 1 and 3 , while the region 2 has a value of $\log (\mathrm{N} / \mathrm{O})=-1.16 \pm 0.23$, respectively.

Figure 12 shows the radial distribution of the oxygen abundance with respect to the peak of $\mathrm{H} \alpha$ emission in the spaxels (red data points) where the [O III] $\lambda 4363$ emission line was 

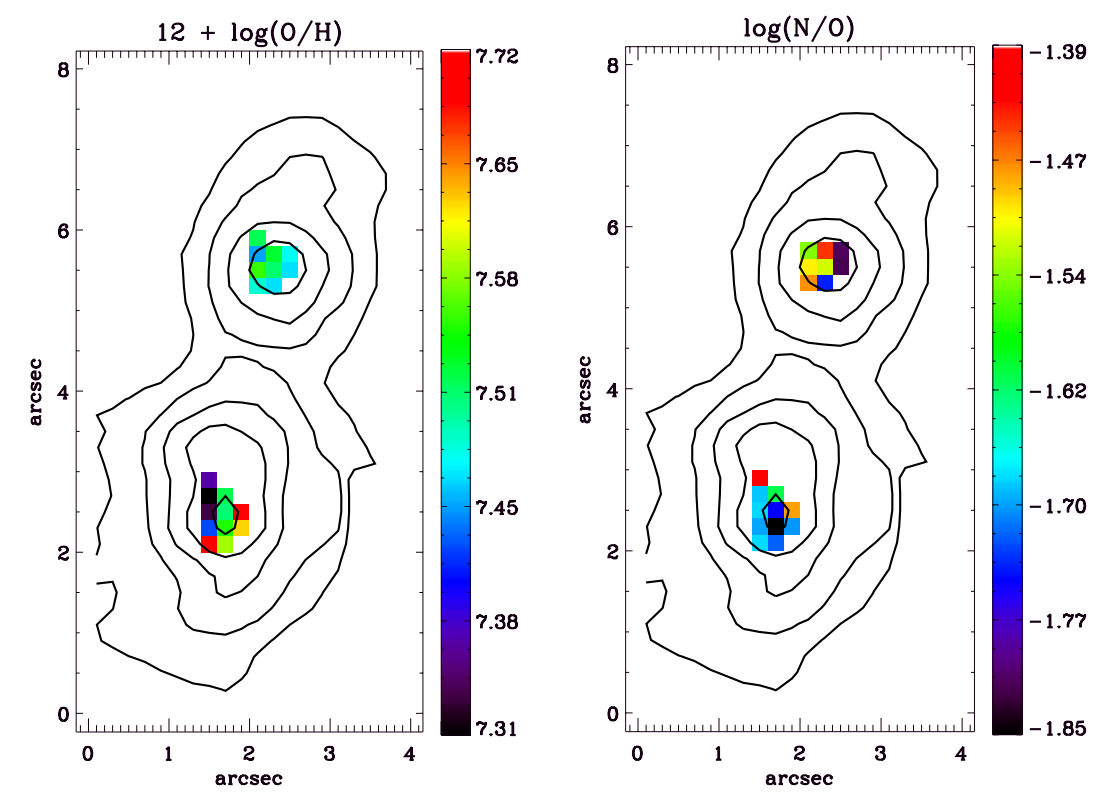

Fig. 11. $12+\log (\mathrm{O} / \mathrm{H})$ abundance and $\log (\mathrm{N} / \mathrm{O})$ ratio maps in spaxels with determined $T_{\mathrm{e}}(\mathrm{O}$ III $)$. Overlaid are the $\mathrm{H} \alpha$ flux contours.

Table 6. Comparison of the chemical properties of regions 1 and 3.

\begin{tabular}{lcccccc}
\hline \hline & \multicolumn{3}{c}{ Region 1 } & \multicolumn{3}{c}{ Region 3 } \\
& Mean & SDEV $^{a}$ & $\left|\Delta^{b}\right|$ & Mean & SDEV & $|\Delta|$ \\
\hline $\mathrm{O}^{+} / \mathrm{H}^{+}\left(\times 10^{5}\right)$ & 0.36 & 0.06 & 0.19 & 0.23 & 0.05 & 0.15 \\
$\mathrm{O}^{++} / \mathrm{H}^{+}\left(\times 10^{5}\right)$ & 2.83 & 0.31 & 0.90 & 3.37 & 1.09 & 3.16 \\
$12+\log (\mathrm{O} / \mathrm{H})$ & 7.50 & 0.04 & 0.11 & 7.54 & 0.14 & 0.41 \\
$\mathrm{~N}^{+} / \mathrm{H}^{+}\left(\times 10^{6}\right)$ & 0.09 & 0.02 & 0.06 & 0.05 & 0.02 & 0.05 \\
$12+\log (\mathrm{N} / \mathrm{H})$ & 5.90 & 0.19 & 0.46 & 5.86 & 0.19 & 0.63 \\
$\log (\mathrm{N} / \mathrm{O})$ & -1.60 & 0.16 & 0.39 & -1.68 & 0.10 & 0.38 \\
\hline
\end{tabular}

Notes. ${ }^{(a)}$ Standard deviation of the spaxels. ${ }^{(b)}$ Difference between minimum and maximum values.

detected. We also include oxygen abundance determinations based on empirical calibrations (black data points in Fig. 12) for spaxels where a reliable determination of $T_{\mathrm{e}}(\mathrm{O}$ III $)$ was not possible. In this case, the $\mathrm{O} / \mathrm{H}$ abundance was derived by applying the relation between the line ratio of $[\mathrm{N} \mathrm{II}] \lambda 6584 / \mathrm{H} \alpha$ with the oxygen abundance from Denicoló et al. (2002), i.e., $12+\log (\mathrm{O} / \mathrm{H})=9.12( \pm 0.05)+0.73( \pm 0.10) \times \mathrm{N} 2$, with $\mathrm{N} 2=$ $\log ([\mathrm{N}$ II $] \lambda 6584 / \mathrm{H} \alpha)$. The average oxygen abundance based on $T_{\mathrm{e}}(\mathrm{O}$ III $)$ was determined to be $12+\log (\mathrm{O} / \mathrm{H})=7.50$ and 7.54 for regions 1 and 3, respectively, slightly above the value from the Denicoló's relation $(\sim 7.48)$, having a typical uncertainty of 0.18 dex. However, the values based on the empirical calibration are, in some cases, by $\sim 0.4$ dex lower than those relying on $T_{\mathrm{e}}(\mathrm{O}$ III), at least in the peak of $\mathrm{H} \alpha$ emission. Therefore, this empirical calibration method provides a useful mean value for the oxygen abundance in the ISM but is less suited for a detailed study of possible spatial variations (e.g., López-Sánchez et al. 2011).

It is interesting to note that the integrated N/O value of the galaxy is similar, within the errors, to the ones obtained in regions 1-3, and those of other BCD galaxies of similar metallicity (see Fig. 13). Indeed, the $\mathrm{O} / \mathrm{H}$ and $\mathrm{N} / \mathrm{O}$ abundances from the integrated spectrum is in excellent agreement, within the errors, with the mean value of the spaxels for regions 1 and 3 . Therefore, our results appear normal for XBCDs, which are characterized

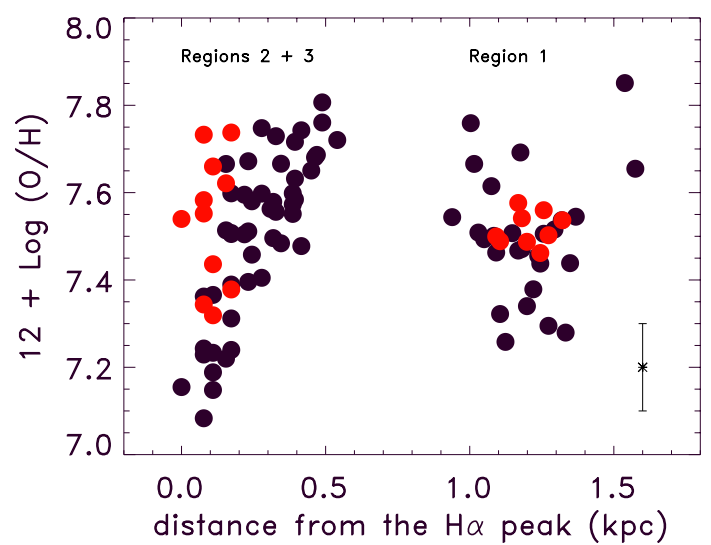

Fig. 12. $12+\log (\mathrm{O} / \mathrm{H})$ radial distribution in HS 2236+1344. Red circles correspond to the data points obtained using the $T_{\mathrm{e}}(\mathrm{O}$ III $)$ and black circles to data points obtained using the Denicoló et al. (2002) calibration. The mean error is shown in the lower of the figure.

by a plateau at $\log (\mathrm{N} / \mathrm{O}) \sim-1.6$ (e.g., Edmunds \& Pagel 1978; Alloin et al. 1979; Izotov \& Thuan 1999).

XBCDs are found to fall off the mass/luminosity - metallicity relation compiled from literature data in Filho et al. (2013), which is consistent with the presence of chemically unpolluted gas in these systems. It is worth remarking in this context that Sánchez almeida et al. (2014) studied, using long-slit spectroscopy, the oxygen abundance along the major axis of seven SF galaxies, including HS $2236+1344$. In that study, they find that the latter shows a central metallicity decrease by $\sim 0.5$ dex, which they ascribe to accretion of metal-poor gas from the halo. It is long known (e.g., Thuan et al. 2005) that the gas in the halos of $\mathrm{H} \mathrm{II} / \mathrm{BCD}$ galaxies is very metal poor, a fact making infall and mixing with gas from the halo a plausible explanation for the reported local decrease in the metallicity of the ionized gas phase. From the present data, however, given the uncertainties associated with our determinations of $\mathrm{O}$ and $\mathrm{N}$, we consider these chemical species to be well mixed and homogeneously distributed over the ISM of the galaxy (e.g., Lagos et al. 2009, 2012), albeit a slightly, and uncertain, decreased 


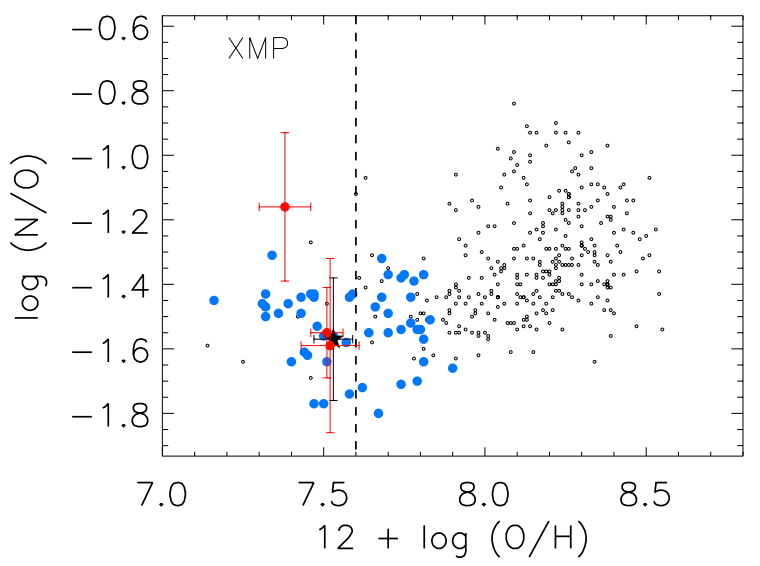

Fig. 13. $\log (\mathrm{N} / \mathrm{O})$ vs. $12+\log (\mathrm{O} / \mathrm{H})$ abundances. The values of the three GH IIRs resolved in HS 2236+1344 are plotted by red points. The black star corresponds with the integrated value of the galaxy. While the small black open dots corresponds with values found by Izotov et al. (2006b) for a large sample of SDSS starburst galaxies and blue dots show data from Izotov et al. (2012) for a sample of XBCDs.

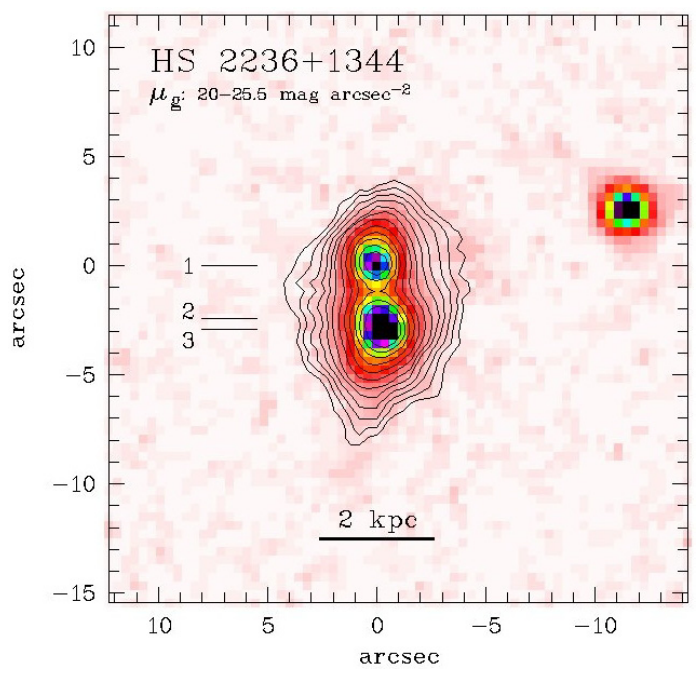

Fig. 14. SDSS $g$ band image of HS $2236+1344$ with overlaid contours between 20 and $25.5 \mathrm{~g} \mathrm{mag} \operatorname{arcsec}^{-2}$ in steps of $0.5 \mathrm{mag}$. The three GH IIRs in the XBCD are labeled.

oxygen abundance was found in the faintest GH IIR studied $(12+\log (\mathrm{O} / \mathrm{H})=7.38 \pm 0.08)$.

\subsection{The surface brightness profile of HS $2236+1344$}

The surface brightness profile of HS 2236+1344 agrees with the detection of an underlying more evolved stellar host with a luminosity-weighted age of $0.5 \ldots 2 \mathrm{Gyr}$ in most XBCDs studied as yet (Papaderos et al. 2008), even though the estimated ages will depend, besides the poorly constrained SFH, also on the importance of radial stellar migration and the associated radial mass filtering effect described in Papaderos et al. (2002).

From the SDSS $g$-band contour map in Fig. 14 it is apparent that the three GH IIRs in HS $2236+1344$ are hosted by a more extended underlying LSB component with a moderately smooth morphology. This LSB host dominates the light for surface brightness levels $\mu_{g} \gtrsim 24 \mathrm{mag} \operatorname{arcsec}^{-2}$ and can be studied using SDSS data down to $\mu_{g} \simeq 25.5 \mathrm{mag} \operatorname{arcsec}^{-2}$. The surface brightness profiles of the XBCD in $g, r$ and $i$ (Fig. 15, upper panel) were derived with method iv by Papaderos et al. (2002)

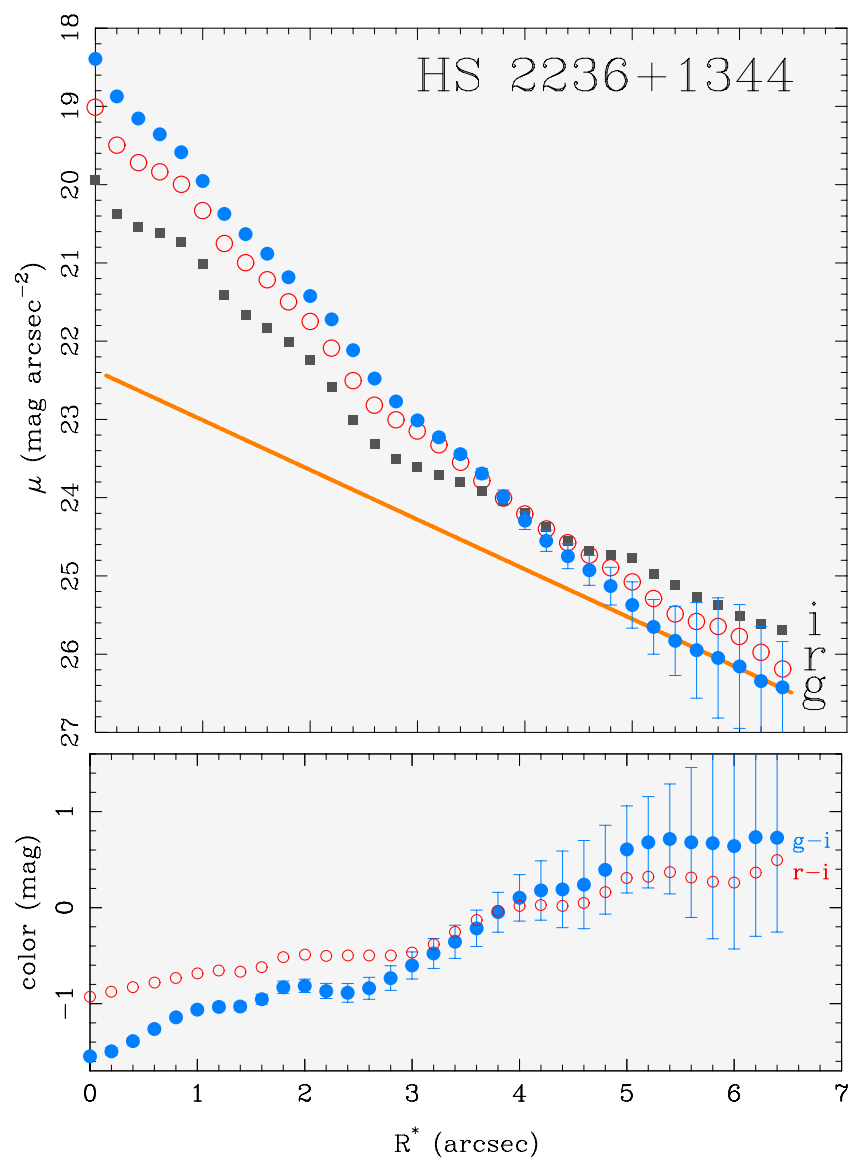

Fig. 15. SDSS $g, r$ and $i$ band surface brightness profiles (upper panel) and $g-i$ and $r-i$ color profiles (lower panel) of HS 2236+1344.

(also referred to as LAZY by Noeske et al. 2006). They show the typically complex radial intensity distribution of BCDs/XBCDs which is characterized by an outer exponential component, corresponding to the LSB host, and a steep luminosity increase at smaller radii that is due to the young stellar population and nebular emission in the starburst component (e.g., Papaderos et al. 1996a). A fit to the $g$ band profile for $R^{\star} \geq 5^{\prime \prime}$, i.e., beyond the transition radius (Papaderos et al. 1996a), where color profiles of BCDs level off to a nearly constant value, yields an exponential scale length of $1.7 \pm 0.1(\simeq 660 \pm 40 \mathrm{pc})$ and a total absolute magnitude of -15.3 mag for the LSB host. The latter contributes only about $15 \%$ of the total luminosity, i.e., significantly less than the average value for normal-metallicity BCDs ( 50\%; Papaderos et al. 1996b). With regard to its effective radius $r_{\mathrm{eff}}$ of $2^{\prime \prime} 2(\simeq 860 \mathrm{pc})$, HS $2236+1344$ does not appear exceptional among BCDs/XBCDs.

The very blue $g-i$ color $(-1.4 \mathrm{mag} \ldots-0.3 \mathrm{mag})$ of the galaxy in its central part (out to $\sim 1.5 r_{\text {eff }}$ ) is only reproducible by a strong nebular emission contribution in the visual passband by the [O III] $\lambda \lambda 4959,5007$ forbidden lines (Papaderos et al. 1998). This is in good agreement with the large measured $\mathrm{H} \beta$ EWs (230...300 A; Table 4) and previous spectroscopic and evolutionary synthesis studies by Guseva et al. (2007) which clearly reveal a strong nebular continuum contribution in the region around the Balmer jump.

The mean $g-i$ color in the LSB component of HS 2236+1344 (of $\sim 0.5 \mathrm{mag}$; Fig. 15, lower panel) corresponds to an age between $\sim 1$ and $\sim 3.3$ Gyr for a SFH approximated, respectively, by an instantaneous burst and an exponentially decreasing star formation rate (SFR) with an e-folding time of 1 Gyr (cf., e.g. Fig. 6 
of Papaderos et al. 2008). Judging from our IFU data, extended ionized gas emission, in this galaxy, does not dominate in the LSB periphery of HS 2236+1344, contrary to the case of I Zw 18 (Papaderos et al. 2002; Papaderos \& Östlin 2012), thus the broadband colours of the LSB need not be corrected for this effect.

If the exponential LSB host of BCDs/XBCDs forms in an inside-out manner through radial migration of stars, then the associated stellar mass filtering effect (Papaderos et al. 2002) will result into a stellar age overestimate, if colours are interpreted in terms of extended SFHs. In fact, a consequence of galaxy build-up through stellar migration is that instantaneous star formation models yield a better approximation to the true stellar age of the LSB host than the usually adopted continuous star formation SFH models (see for an update and a further discussion Papaderos \& Östlin 2012).

\subsection{Star formation and the age of the current burst in HS 2236+1344}

The H $\alpha$ flux in Table 3 translate by the adopted distance and the Kennicutt (1998) conversion formula, $\operatorname{SFR}\left(M_{\odot} \mathrm{yr}^{-1}\right)=$ $5.3 \times 10^{-42} L(\mathrm{H} \alpha) \mathrm{erg} \mathrm{s}^{-1}$, after correction for a Kroupa IMF (Calzetti et al. 2007) to an integrated SFR of $0.587 M_{\odot} \mathrm{yr}^{-1}$. The SFRs in regions $1-3$ are estimated to be $\simeq 0.075,0.124$ and $0.216 M_{\odot} \mathrm{yr}^{-1}$, respectively. The corresponding SFR per unit of area $\left(\Sigma_{\mathrm{SFR}}\right)$ is then $\sim 0.629, \sim 0.695$ and $\sim 1.810 M_{\odot} \mathrm{yr}^{-1} \mathrm{kpc}^{-2}$, by some factors greater than the integrated value of the galaxy $\left(\sim 0.145 M_{\odot} \mathrm{yr}^{-1} \mathrm{kpc}^{-2}\right)$. Note that the $\Sigma_{\mathrm{SFR}}$ in individual regions are comparable to values determined for higher-metallicity starburst galaxies $\left(>0.1 M_{\odot} \mathrm{yr}^{-1} \mathrm{kpc}^{-2}\right.$; Daddi et al. 2010) and greater than typical values of normal spirals $\left(\Sigma_{\mathrm{SFR}}<\right.$ $0.1 M_{\odot} \mathrm{yr}^{-1} \mathrm{kpc}^{-2}$; Kennicutt 1998). The integrated $\Sigma_{\mathrm{SFR}}$ in HS $2236+1344$ is also greater than the ones found in other BCD galaxies, such as Mrk 36 and UM 4610.039 and $0.052 M_{\odot} \mathrm{yr}^{-1} \mathrm{kpc}^{-2}$ (Lagos et al. 2011), respectively, and local cometary/tadpole SF galaxies $\left(\sim 0.01 M_{\odot} \mathrm{yr}^{-1} \mathrm{kpc}^{-2}\right.$; Elmegreen et al. 2012), yet comparable to the SFR in 30 Doradus (0.36 $M_{\odot} \mathrm{yr}^{-1} \mathrm{kpc}^{-2}$; Chen et al. 2005).

Following common practice, an estimate of the starburst age in HS 2236+1344 can be obtained from comparison of the observed $\mathrm{EW}(\mathrm{H} \alpha)$ and $\mathrm{EW}(\mathrm{H} \beta)$ distribution with predictions from zero-dimensional evolutionary synthesis models. For this, we use Starburst99 models (Leitherer et al. 1999) for a metallicity $Z=0.004$, Geneva evolutionary stellar tracks, and a Kroupa initial mass function $\left(\propto M^{-1}\right)$ with $\alpha=1.3$ for stellar masses between 0.1 to $0.5 M_{\odot}$ and $\alpha=2.3$ between 0.5 and $100 M_{\odot}$. For the SFH we consider the limiting cases of a single burst and continuous star formation with a constant SFR of 0.075 and $0.170 M_{\odot} \mathrm{yr}^{-1}$ for regions 1 and $2+3$, respectively. The stellar age, in the FoV, obtained from Starburst 99 models for these two SFHs (Fig. 16) is very low ( 3 3 . 6 Myr) for the instantaneous burst model, and between $\sim 4$ and $\sim 100$ Myr for continuous star formation. We note that the assumed metallicity $(Z=0.004)$ does not significantly affect the derived age, given that we obtain nearly the same age pattern when we assume a metallicity of $Z=0.001$. Figure 16 suggests that regions 1 and $2+3$ were formed almost coevally some $3 \mathrm{Myr}$ ago, assuming an instantaneous burst, whereas their surroundings and the arm-like features appear to be slightly older. It should be born in mind that the EW maximum of nebular emission lines in starburst galaxies does not necessarily spatially coincide with the location of ionizing YSCs (e.g., Papaderos et al. 1998, 2002; Guseva et al. 2004; Lagos et al. 2007; Papaderos \& Östlin 2012), consequently
EW maps are not always convertible into stellar age maps via standard evolutionary synthesis models (e.g., Starburst99). The unknown SFH is another important source of uncertainty in converting $\mathrm{H} \alpha$ luminosities into SFRs for starburst galaxies (e.g., Weilbacher \& Fritze von Alvensleben 2001), just like the fraction of ionizing Lyman continuum photons leaking out of SF regions and galaxies (e.g., see also Guseva et al. 2007; Relaño et al. 2012; Papaderos et al. 2013; Bergvall et al. 2013). Therefore, the estimates above are indicative only, and are merely provided for the sake of comparison with other studies of local SF galaxies.

We reiterate that several studies point to similar physical conditions across the ISM of XBCD (P12), H II/BCDs (e.g., UM 408, and Tol 0104-388 and Tol 2146-391; Lagos et al. 2009,2012 ) and to nearly coeval SF activity in these systems on spatial scales of $\sim 1 \mathrm{kpc}$ (e.g., Lagos et al. 2011). Whereas most low-luminosity BCDs show a smooth morphology in their LSB host, luminous ( $M_{B} \lesssim-19 \mathrm{mag}$ ) ones often show tidal distortions on deep images (e.g., Bergvall \& Östlin 2002; Lagos et al. 2007), suggesting strong interactions or merging as the primary triggering agent of their starburst activity (e.g., Taylor et al. 1995; Pustilnik et al. 2001a; Brosch et al. 2004; Ekta et al. 2008). If SF activity in HS $2236+1344$ is due to a recent or ongoing merger, as suggested by Moiseev et al. (2010), a flatter metallicity distribution may be evolved through transport and mixing of metals (e.g., Rupke et al. 2010; Montuori et al. 2010) following tidal tail formation. Thus far, in most studies on chemical abundances in SF dwarf galaxies (e.g., Lee \& Skillman 2004; Kehrig et al. 2008; Lagos et al. 2009, 2012) there are no clear evidences of abundance variations. Therefore, the overall chemical homogeneity of the warm ISM in HS 2236+1344 suggests an efficient dispersal and mixing of heavy elements, albeit a slightly and uncertain decreased oxygen abundance was found in region 2 . In any case, we emphasize that given the uncertainties, in this study, we must consider the abundances across the galaxy as fairly homogeneous. On the other hand, the estimated age of the GH IIRs, found in this galaxy, suggests that the current, large-scale, burst started recently and likely simultaneously. Therefore, the triggering mechanism (i.e., minor interactions or infall of gas from the halo) may be related to the overall physical conditions of the ISM, particularly the gas surface density, in conjunction with small stochastic effects.

Clearly, detailed studies of the ISM with IFU spectroscopy (see, e.g., Lagos \& Papaderos 2013) have the potential of greatly improving our understanding of the evolution of chemical abundance patterns and star formation in $\mathrm{HII} / \mathrm{BCD}$ galaxies.

\section{Summary}

Our spatially resolved study of the warm ISM in the XBCD galaxy HS $2236+1344$ by means of IFU spectroscopy yields the following conclusions:

1. HS $2236+1344$ contains three GH IIRs with an $\mathrm{H} \alpha$ luminosity in the range $\sim 1 \ldots 4 \times 10^{40} \mathrm{erg} / \mathrm{s}$. The high angular resolution acquisition image of the galaxy additionally shows some faint arm-like structures in the close vicinity of the northern and southern GH IIR, which might be of tidal origin or due to expanding gas shells. The structure of the ionized gas, as traced both by the emission lines and the continuum are fairly similar.

2. The $\mathrm{H} \alpha$ velocity field $v_{r}(\mathrm{H} \alpha)$ of the galaxy shows a smooth gradient along its major axis, with a difference of about $80 \mathrm{~km} \mathrm{~s}^{-1}$ between its receding northwestern and approaching southeastern half. The observed velocity range 

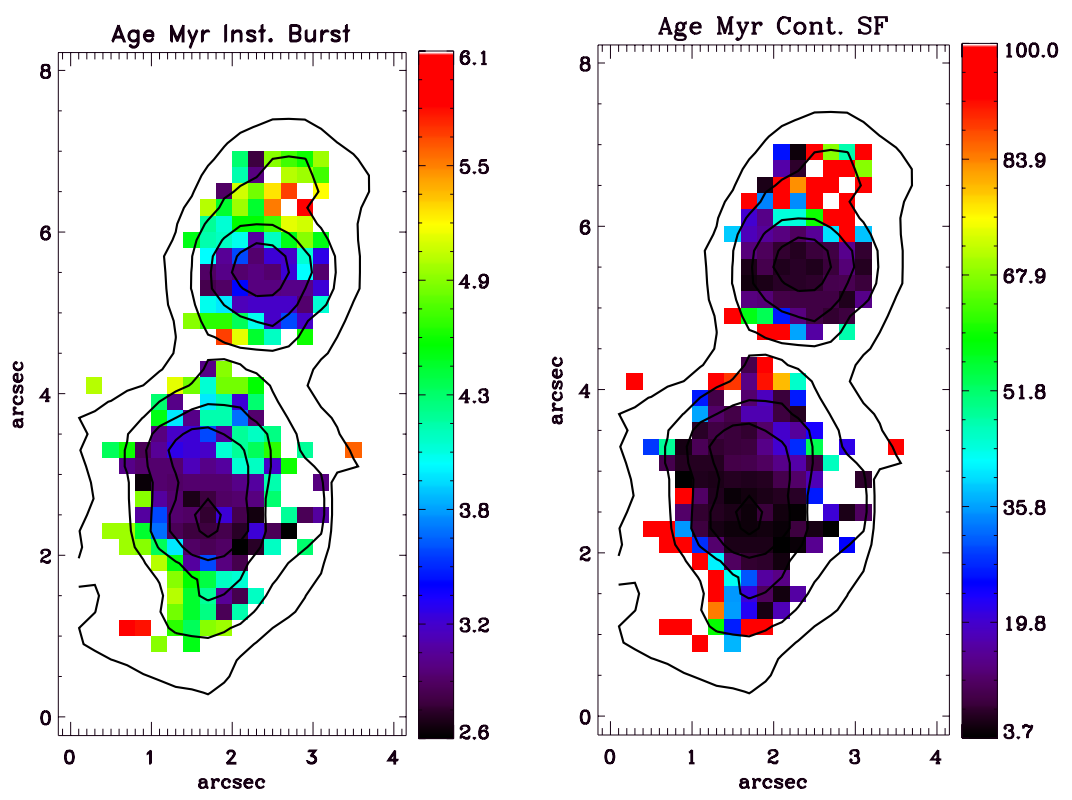

Fig. 16. Age distribution in Myr obtained by direct conversion of $\mathrm{EW}(\mathrm{H} \alpha)$ and $\mathrm{EW}(\mathrm{H} \beta)$ maps into stellar ages using Starburst99 model predictions for an instantaneous burst (left panel) and continuous SF (right panel). Overlaid are the $\mathrm{H} \alpha$ flux contours.

is comparable to values determined for other BCDs (e.g., van Zee et al. 2001).

3. A comparison of $\mathrm{EW}(\mathrm{H} \alpha)$ and $\mathrm{EW}(\mathrm{H} \beta)$ maps with predictions from the evolutionary synthesis code Starburst99 suggests that the three GH IIRs in HS 2236+1344 were formed almost coevally less than $\sim 3$ Myr ago. The estimated SFR surface density of $\Sigma_{\mathrm{SFR}} \sim 0.2 M_{\odot} \mathrm{yr}^{-1} \mathrm{kpc}^{-2}$ is greater than the ones found in other low-luminosity $\mathrm{H} \mathrm{II} / \mathrm{BCD}$ galaxies (Lagos et al. 2011) and local cometary/tadpole SF galaxies (Elmegreen et al. 2012). Surface brightness profiles, derived from SDSS data, reveal a compact underlying stellar host, which presumably indicates that HS 2236+1344 has undergone previous SF activity, as was found to be the case in most XMP BCDs studied as yet (e.g., Papaderos et al. 2008).

4. The high-ionization He II $\lambda 4686$ emission line is detected in HS 2236+1344. Similar to many BCDs with He II $\lambda 4686$ emission, HS 2236+1344 shows no WR bump. The spatial distribution of this high-ionization emission, observed in this study, suggests that H II $\lambda 4686$ in HS 2236+1344 is associated with the current burst of star formation.

5. We calculated the spatial distribution of oxygen and nitrogen, and their ratio, based on spaxels with reliable $T_{\mathrm{e}}(\mathrm{O}$ III) determinations. The oxygen abundance in the three resolved GH IIRs is consistent, within uncertainties, with the integrated value of $12+\log (\mathrm{O} / \mathrm{H})=7.53 \pm 0.06$, suggesting a nearly constant metallicity across the ISM of HS 2236+1344. With regard to the nitrogen-to-oxygen abundance ratio, we derive a value of $\log (\mathrm{O} / \mathrm{N})=-1.57 \pm$ 0.19 for the integrated spectrum, in good agreement with $\mathrm{N} / \mathrm{O}$ determinations for other XBCDs $(\log (\mathrm{N} / \mathrm{O}) \simeq-1.6)$. The overall chemical homogeneity of the warm ISM in HS 2236+1344 suggests, in line with previous studies, an efficient dispersal and mixing of heavy elements in the lowestmetallicity $\mathrm{H} \mathrm{II/BCD}$ galaxies.

Acknowledgements. We would like thank the anonymous referee for the comments and suggestions that substantially improved the paper. P.L. is supported by a Postdoctoral grant SFRH/BPD/72308/2010, funded by Fundação para a Ciência e a Tecnologia (FCT). P.P. is supported by Ciencia 2008 Contract, funded by FCT/MCTES (Portugal) and POPH/FSE (EC). J.M.G. is supported by a Postdoctoral grant SFRH/BPD/66958/2009, funded by FCT (Portugal). P.L., P.P. and J.M.G. acknowledge support by the FCT under project FCOMP-01-0124-FEDER-029170 (Reference FCT PTDC/FISAST/3214/2012), funded by the FEDER program. A.V.S.C. acknowledge financial support from Consejo Nacional de Investigaciones Científicas y Técnicas, Agencia Nacional de Promoción Científica y Tecnológica (PICT 2010-0410), and Universidad Nacional de La Plata (Argentina). This research has made use of the NASA/IPAC Extragalactic Database (NED) which is operated by the Jet Propulsion Laboratory, California Institute of Technology, under contract with the National Aeronautics and Space Administration. Based on observations obtained at the Gemini Observatory (Program ID: GN-2010B-Q-69), which is operated by the Association of Universities for Research in Astronomy, Inc., under a cooperative agreement with the NSF on behalf of the Gemini partnership: the National Science Foundation (United States), the Science and Technology Facilities Council (United Kingdom), the National Research Council (Canada), CONICYT (Chile), the Australian Research Council (Australia), Ministério da Ciência e Tecnologia (Brazil) and Ministerio de Ciencia, Tecnología e Innovación Productiva (Argentina).

\section{References}

Adamo, A., Östlin, G., Zackrisson, E., et al. 2011, MNRAS, 415, 2388 Allington-Smith, J., Graham, M., Content, R., et al. 2002, PASP, 114, 892

Alloin, D., Collin-Souffrin, S., Joly, M., \& Vigroux, J. M., 1979, A\&A, 78, 200 Amorín, R., Aguerri, J. A., Muñoz-Tuñón, C., \& Cairós, L. M. 2009, A\&A, 501, 75

Amorín, R., Pérez-Montero, E., Vílchez, J. M., \& Papaderos, P. 2012, ApJ, 749, 185

Baldwin, J. A., Phillips, M. M., \& Terlevich, R. 1981, PASP, 93, 5

Bergvall, N. 2012, in Dwarf Galaxies: Keys to Galaxy Formation and Evolution, Proc. of JENAM 2010 (Lisbon, September 2010), eds. P. Papaderos, S. Recchi, \& G. Hensler (Springer Verlag), 175 Bergvall, N., \& Östlin, G. 2002, A\&A, 390, 891

Bergvall, N., Leitet, E., Zackrisson, E., Marquart, T. 2013, A\&A, 554, A38

Brinchmann, J., Kunth, D., \& Durret, F. 2008, A\&A, 485, 657

Brosch, N., Almoznino, E., \& Heller, A. B. 2004, MNRAS, 349, 357

Cairós, L. M., Caon, N., González-Pérez, J. N., et al. 2001, ApJS, 133, 321

Cairós, L. M., Caon, N., Zurita, C., et al. 2009, A\&A, 507, 1291

Cairós, L. M., Caon, N. García Lorenzo, B., et al. 2012, A\&A, 547, A24

Calzetti, D., et al. 2007, ApJ, 666, 870

Cardamone, C., Schawinski, K., Sarzi, M., et al. 2009, MNRAS, 399, 1191

Cardelli, J. A., Clayton, G. C., \& Mathis, J. S., 1989, ApJ, 345, 245

Chen, C.-H. R., Chu, Y.-H., \& Johnson, K. E. 2005, ApJ, 619, 779

Daddi, E., Elbaz, D., Wlater, F., et al. 2010, ApJ, 714, L118 
Denicoló, G., Terlevich, R. \& Terlevich, E. 2002, MNRAS, 330, 69 De Robertis, M. M., Dufour, R. J., \& Hunt, R. W. 1987, JRASC, 81, 195 Dopita, M. A., \& Sutherland R. S. 1996, ApJS, 102, 161

Edmunds, M. G., \& Pagel, B. E. J. 1978, MNRAS, 185, 77

Ekta, B., Chengalur, J. N., \& Pustilnik, S. A. 2008, MNRAS, 391, 881 Elmegreen, D. M., Elmegreen, B. G., Rubin, D. S., et al. 2005, ApJ, 631, 85 Elmegreen, D. M., Elmegreen, B. G., Ravindranath, S., et al. 2007, ApJ, 658, 763

Elmegreen, D. M., Elmegreen, B. G., Sánchez Almeida, J., et al. 2012, ApJ, 750, 95

Filho, M., Winkel, B., Sánchez Almeida, J., et al. 2013, A\&A, 558, A18

Gil de Paz, A., Madore, B. F., \& Pevunova, O. 2003, ApJS, 147, 29

Guseva, N. G., Izotov, Y. I., \& Thuan, T. X. 2000, ApJ, 531, 776

Guseva, N. G., Papaderos, P., Izotov, Y. I., Noeske, K. G., \& Fricke, K. J. 2004, A\&A, 421, 519

Guseva, N. G., Izotov, Y. I., Papaderos, P., \& Fricke, K. J. 2007, A\&A, 464, 885

Guseva, N. G., Papaderos, P., Meyer, H. T., Izotov, Y. I., \& Fricke, K. J. 2009, A\&A, 505, 63

Guzmán, R., Jangren, A., Koo, D. C., Bershady, M. A., \& Simard, L. 1998, ApJ, 495, L13

Hook, I., Jørgensen, I., Allington-Smith, J. R., et al. 2004, PASP, 116, 425

Izotov, Y. I., \& Thuan T. X. 1999, ApJ, 511, 639

Izotov, Y. I., \& Thuan T. X. 2007, AJ, 665, 1115

Izotov, Y. I., \& Thuan T. X. 2009, ApJ, 690, 1797

Izotov, Y. I., Dyak, A. B., Chaffee, F. H., et al. 1996, ApJ, 458, 524

Izotov, Y. I., Lipovetsky, V. A., Chaffee, F. H., et al. 1997a, ApJ, 476, 698

Izotov, Y. I., Foltz, C. B., Green, R. F., Guseva, N. G., \& Thuan, T. X. 1997b, ApJ, 487, 37

Izotov, Y. I., Thuan, T. X., \& Guseva, N. G. 2005, ApJ, 632, 210

Izotov, Y. I., Schaerer, D., Blecha, A., et al. 2006a, A\&A, 459, 71

Izotov, Y. I., Papaderos, P., Guseva, N. G., Fricke, K. J., \& Thuan, T. X. 2006b, A\&A, 454, 137

Izotov, Y. I., Guseva, N. G., Fricke, K. J., \& Papaderos, P. 2009, A\&A, 503, 61

Izotov, Y. Y., Thuan, T. X., \& Guseva, N. G. 2012, A\&A, 546, A122

Kehrig, C., Vílchez, J. M., Sánchez, S. F., et al. 2008, A\&A, 477, 813

Kehrig, C., Pérez-Montero, E., Vílchez, J. M., et al. 2013, MNRAS, 432, 2731

Kennicutt, R. C. 1998 ARA\&A, 36, 189

Kewley, L. J., Dopita, M. A., Sutherland, R. S., Heisler, C. A., \& Trevena, J. 2001, ApJ, 556, 121

Kniazev, A. Y., Pustilnik, S. A., Grebel, E. K., Lee, H., \& Pramskij, A. G. 2004, ApJS, 153, 429

Kobulnicky, H. A., \& Skillman, E. D. 1997, ApJ, 489, 636

Kobulnicky, H. A., Skillman, E. D., Roy, J.-R., Walsh, J. R., \& Rosa M. R. 1997, ApJ, 477, 679

Krabbe, A. C., Rosa, D. A., Dors, O. L., et al. 2014, MNRAS, 437, 1155

Kunth, D., \& Sargent, W. L. W. 1983, ApJ, 273, 81

Kunth, D., \& Sargent, W. L. W. 1986, ApJ, 300, 496

Kunth, D., \& Östlin, G. 2000, A\&ARv, 10, 1

Lagos, P., \& Papaderos, P. 2013, Adv. Astron., 2013, 20

Lagos, P., Telles, E., \& Melnick, J. 2007, A\&A, 476, 89

Lagos P., Telles E., Muñoz-Tuñón, C., et al. 2009, AJ, 137, 5068

Lagos, P., Telles, E., Nigoche-Netro, A., \& Carrasco, E. R. 2011, AJ, 142, 162

Lagos, P., Telles, E., Nigoche-Netro, A., \& Carrasco, E. R. 2012, MNRAS, 427, 740

Lee, H., \& Skillman, E. D. 2004, ApJ, 614, 698

Leitherer, C., Schaerer, D., Goldader, J. D., et al. 1999, ApJS, 123, 3

Lequeux, J., \& Viallefond, F. 1980, A\&A, 91, 269

Loose, H.-H., \& Thuan, T. X. 1986, ApJ, 309, 59

López-Sánchez, A. R., Mesa-Delgado, A., López-Martín, L., \& Esteban, C. 2011, MNRAS, 411, 2076

López-Hernández, J., Terlevich, E., Terlevich, R., et al. 2013, MNRAS, 430, 472

Moiseev, A. V., Pustilnik, S. A., \& Kniazev, A. Y. 2010, MNRAS, 405, 2453
Montuori, M., Di Matteo, P., Lehnert, M. D., Combes, F., \& Semelin, B. 2010, A\&A, 518, A56

Morales-Luis, A. B., Sánchez Almeida, J., Aguerri, J. A. L., \& Muñoz-Tuñón, C. 2011, ApJ, 743, 77

Noeske, K. G., Iglesias-Páramo, J., Vílchez, J. M., Papaderos, P., \& Fricke, K. J. 2001, A\&A, 371, 806

Noeske, K. G., Koo. D. C. Phillips, A. C., et al. 2006, ApJ, 640, 143

Osterbrock, D. E., \& Ferland, G. J. 2006, Astrophysics of gaseous nebulae and active galactic nuclei, 2nd. edn. (Sausalito, CA: University Science Books)

Östlin, G., Zackrisson, E., Bergvall, N., \& Rönnback, J. 2003, A\&A, 408, 887

Papaderos, P. 2012, in Dwarf Galaxies: Keys to Galaxy Formation and Evolution, Proc. of JENAM 2010 (Lisbon, September 2010), eds. P. Papaderos, S. Recchi, \& G. Hensler (Springer Verlag), 321

Papaderos, P., \& Östlin, G. 2012, A\&A, 537, A126

Pagel, B. E. J., Simonson E. A., Terlevich R. J., \& Edmunds, M. G. 1992, MNRAS, 255, 325

Papaderos, P., Loose, H.-H., Thuan, T. X., \& Fricke, K. J. 1996a, A\&AS, 120, 207

Papaderos, P., Loose, H.-H., Fricke, K. J., \& Thuan, T. X. 1996b, A\&A, 314, 59

Papaderos, P., Izotov, Y. I., Fricke, K. J., Thuan, T. X., \& Guseva, N. G. 1998, A\&A, 338, 43

Papaderos, P., Izotov, Y. I., Thuan, T. X., et al. 2002, A\&A, 393, 461

Papaderos, P., Izotov, Y. I., Guseva, N. G., Thuan, T. X., \& Fricke, K. J. 2006, A\&A, 454, 119

Papaderos, P., Guseva, N. G., Izotov, Y. I., \& Fricke, K. J. 2008, A\&A, 491, 113

Papaderos, P., Gomes, J. M., Vílchez, J. M., et al. 2013, A\&A, 555, A1

Pérez-Montero, E., Vílchez, J. M., Cedrés, B., et al. 2011, A\&A, 532, 141

Pustilnik, S. A., \& Martin, J.-M. 2007, A\&A, 464, 859

Pustilnik, S. A., Kniazev, A. Y., Lipovetsky, V. A., \& Ugryumov, A. V. 2001a, A\&A, 373, 24

Pustilnik, S. A., Brinks, E., Thuan, T. X., Lipovetsky, V. A., \& Izotov, Y. I. 2001b, AJ, 121, 1413

Pustilnik, S., Kniazev, A., Pramskij, A., et al. 2004, A\&A, 419, 469

Relaño, M., Kennicutt, R. C., Jr., Eldridge, J. J., Lee, J. C., \& Verley, S. 2012, MNRAS, 423, 2933

Rupke, D. S. N., Kewley, L. J., \& Barnes, J. E. 2010, ApJ, 710, 156

Sánchez Almeida, J., Morales-Luis, A. B., Muñoz-Tuñón, C., et al. 2014, ApJ, 783,45

Shirazi, M., \& Brinchmann, J. 2012, MNRAS, 421, 1043

Skillman, E. D. 2012, in Dwarf Galaxies: Keys to Galaxy Formation and Evolution, Proc. JENAM 2010 (Lisbon, September 2010), eds. P. Papaderos, S. Recchi, \& G. Hensler, (Springer Verlag), 3

Straughn, A. N., Cohen, S. H., Ryan, R. E., et al. 2006, ApJ, 639, 724

Taylor, C. L., Brinks, E., Grashuis, R. M., \& Skillman, E. D. 1995, ApJS, 99, 427

Telles, E. 2010, ASPC, 423, 65

Telles, E., \& Maddox, S. 2000, MNRAS, 311, 307

Telles, E., \& Terlevich, R. 1997, MNRAS, 286, 183

Telles, E., Melnick, J., \& Terlevich, R. 1997, MNRAS, 288, 78

Tenorio-Tagle, G. 1996, AJ, 111, 1641

Terlevich, R., Melnick, J., Masegosa, J., Moles, M., \& Copetti, M. V. F. 1991, A\&AS, 91, 285

Thuan, T. X., \& Izotov, Y. I. 2005, ApJS, 161, 240

Thuan, T. X., \& Martin, G. E. 1981, ApJ, 247, 823

Thuan, T. X., Lecavelier des Etangs, A., \& Izotov, Y. I. 2005, ApJ, 621, 269 van den Bergh, S., Abraham, R. G., \& Ellis, R. S., et al. 1996, AJ, 112, 359 van Zee, L., Salzer, J. J., \& Skillman, E. D. 2001, AJ, 122, 121

Walsh, J. R., \& Roy, J. R. 1989, MNRAS, 239, 297

Weilbacher, P. M., \& Fritze-v. Alvensleben, U. 2001, A\&A, 373, L9

Windhorst, R. A., Cohen, S. H., Straughn, A. N., et al. 2006, New Astron. Rev., 50,821 